\title{
Transient dynamics of turbulence growth and bursting: Effects of drag- reducing polymers
}

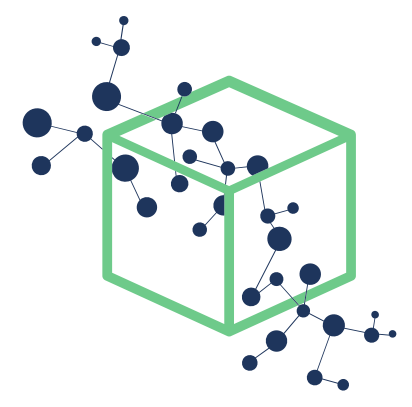

Zhu, Bai, Krushelnycky, and Xi (2019)

XI RESEARCH

DOI: 10.1016/j.jnnfm.2019.03.002

http://xiresearch.org

This document is the accepted manuscript (after peer review) version of an article published in its final form (i.e., the version of record) by Elsevier as

Lu Zhu, Xue Bai, Evan Krushelnycky, and Li Xi. Transient dynamics of turbulence growth and bursting: Effects of drag-reducing polymers. Journal of Non-Newtonian Fluid Mechanics, 266:

127-142, 2019. doi: 10.1016/j.jnnfm.2019.03.002

(copyright $(2019$, Elsevier). The version of record is hosted at

https://dx.doi.org/10.1016/j.jnnfm.2019.03.002

by the publisher.

The current version is made available under the CC-BY-NC-ND 4.0 license (https://creativecommons. org/licenses/by-nc-nd/4.0/) in accordance with the publisher's policy. Please refer to the publisher's site for additional terms of use.
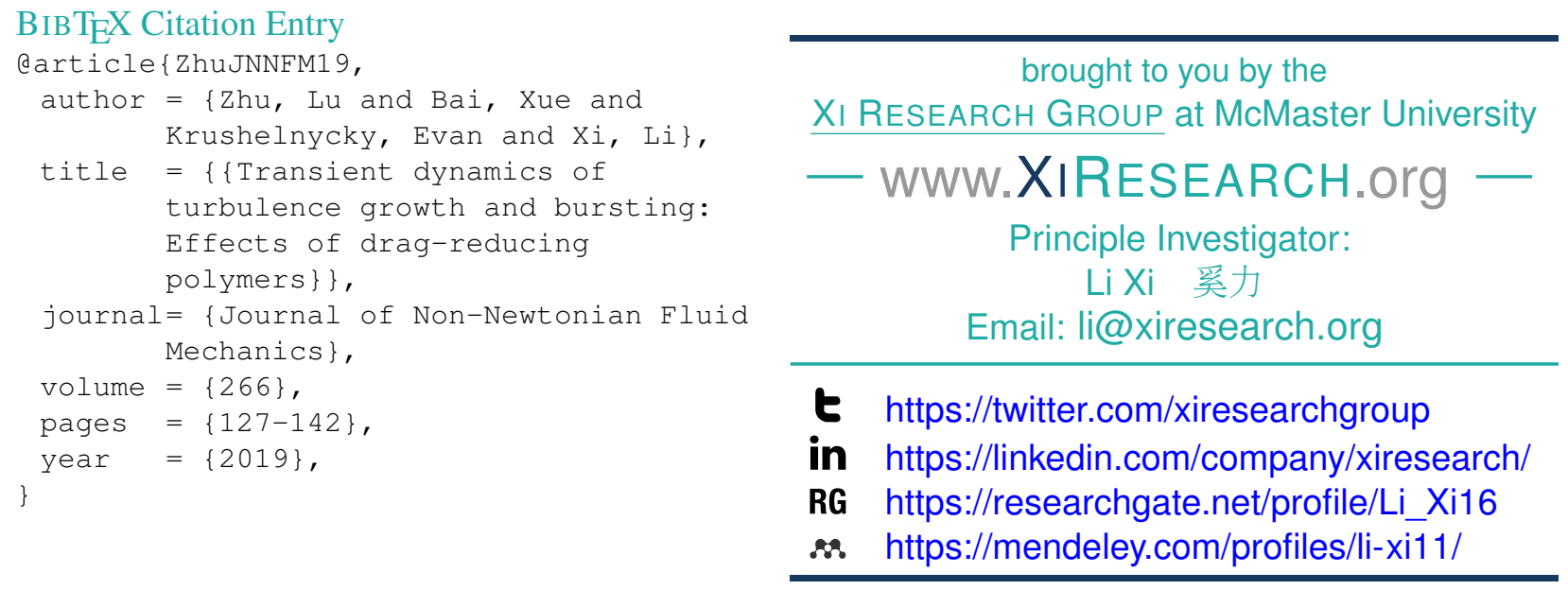


\title{
Transient dynamics of turbulence growth and bursting: effects of drag-reducing polymers
}

\author{
Lu Zhu ${ }^{\mathrm{a}}$, Xue Bai ${ }^{\mathrm{a}}$, Evan Krushelnycky ${ }^{\mathrm{a}}, \mathrm{Li} \mathrm{Xi}^{\mathrm{a}, \mathrm{b}, *}$ \\ ${ }^{a}$ Department of Chemical Engineering, McMaster Universtiy, Hamilton, Ontario L8S \\ 4L7, Canada \\ ${ }^{b}$ Kavli Institute for Theoretical Physics (KITP), University of California, Santa \\ Barbara, California 93106-4030, U.S.A
}

\begin{abstract}
The transient process of turbulence development and vortex breakdown from a marginal flow state dominated by streaky velocity patterns is not only essential for understanding the bypass transition into turbulence, but - in the context of viscoelastic fluids - also offers unique insight into the dynamics at high-extent and maximum drag reduction (HDR and MDR). Shooting trajectories connecting the edge state and following its unstable manifold to the turbulent basin are generated. In Newtonian flow, the growth of turbulence starts with the intensification of velocity streaks and a sharp rise in the Reynolds shear stress. It is followed by a quick breakdown into highintensity small-scale fluctuations before entering the basin of statistical turbulence. Adding drag-reducing polymers does not affect the initial growth of turbulence but stabilizes the primary streak-vortex structure. As a result, the vortex breakdown stage is circumvented. Polymer deformation is insignificant until the vortex breakdown, after which polymer stress rapidly
\end{abstract}

*corresponding author, E-mail: xili@mcmaster.ca 
shoots up. At high Weissenberg number Wi, loss of turbulent kinetic energy through polymer elastic conversion is comparable to viscous dissipation. Beyond bypass transition, the transient process studied here closely resembles the bursting phase of the self-sustaining cycle of turbulence. Our results indicate that at high Wi (i.e., HDR) polymer effects can significantly reduce bursting by rerouting the trajectory of turbulent dynamics.

Keywords:

laminar-turbulent transition, turbulent drag reduction, viscoelastic fluids, direct numerical simulation, flow instability

\section{Introduction}

The transition from a laminar flow to turbulence is accompanied by an abrupt increase in the friction drag of the flow. Long-chain flexible polymer additives are known to cause significant drag reduction (DR) even at very low concentrations $[1,2,3,4]$. Much progress has been made in the fundamental understanding of the DR phenomenon since its initial discovery in the 1940s [5], especially during the past 20 years when tools providing direct access to turbulent flow fields, including particle image velocimetry (PIV) $[6,7]$ and direct numerical simulations (DNS) [8, 9], became broadly applied. In particular, although the exact mechanism of DR remains debatable [3], it is now generally accepted that polymers suppress turbulence by counteracting the vortical motions therein $[10,11,12,13]$. Dynamics of viscoelastic turbulence is however a complex multistage process [14]. Its behaviors remain puzzling in several regimes including that of high DR levels and during the laminar-turbulent (L-T) transition. 
The level of DR initially increases with polymer-induced elasticity but eventually saturates into an asymptotic limit [1]. This limit, the so-called maximum drag reduction (MDR) asymptote, is widely regarded as the most important unsolved problem in viscoelastic turbulence. Mean velocity at MDR is insensitive to polymer species, molecular weight, or concentration and is well approximated by the Virk [1] profile

$$
U_{\mathrm{m}}^{+}=11.7 \ln y^{+}-17.0
$$

(the superscript "+" denotes quantities non-dimensionalized in turbulent inner scales[15]; further explained below in Section 2). The logarithmic relation was empirically proposed without any physical ansatz, other than following the form of the Prandtl-von Kármán (PvK) log law of Newtonian turbulence [15]. Recent studies by White et al. [16] and Elbing et al. [17] closely examined the mean velocity of channel and boundary layer flows using data from both experiments and Direct Numerical Simulation (DNS), which clearly showed that at least for the friction Reynolds number $\operatorname{Re}_{\tau}$ in the range of $O\left(10^{2} \sim 10^{3}\right)$, a pronounced log law region only exists in Newtonian and low-extent drag reduction (LDR) flows - as the flow converges to MDR, logarithmic dependence is lost across the domain. Equation (1) appears close to MDR mean velocity profiles only because any departure from the logarithmic dependence is not easily detectable by eye in common $U_{\mathrm{m}}^{+}$ vs. $y^{+}$linear-log coordinates (as in fig. 11). It should therefore only be interpreted as nothing more than a quantitative estimate for MDR mean velocity: for a very broad range of Reynolds number Re, it provides a reasonable fit for the mean velocity data of various polymer solutions and flow conditions. Qualitative understanding of the existence and universality of MDR are at 
the heart of decoding MDR. The existence of MDR, that polymers are not able to completely quench turbulence but only push it towards an asymptotic limit, indicates that there must be a unique mechanism for sustaining turbulence. This is further supported by the observation of distinct flow structures at MDR compared with those of Newtonian turbulence $[6,7,18,19,20]$. Its universality - for given Re, the same MDR mean velocity is observed for different polymer solutions - is highly counter-intuitive, as MDR is typically reached at the limit of strong polymer effects. Earlier theoretical attempts at explaining MDR are phenomenological or semi-empirical in nature [1, 21, 22] and have met contradictory evidences as new experimental and numerical data emerged (see discussion in White and Mungal [3], Xi and Graham [23].) A consistent mechanistic theory is still missing. It was recognized more recently that the mean velocity profile takes notably different shape way before MDR $[24,19,14,16,17]$ and the concept of high-extent DR (HDR) was thus introduced. The change between LDR and HDR was later shown to be a qualitative transition marked by sharp changes in several key flow statistics and different vortex distribution patterns, which indicates fundamental changes in the underlying vortex regeneration dynamics [25].

As to the L-T transition, since polymers can suppress turbulent fluctuations, it is intuitive to expect that the transition will be delayed to a higher Re compared with Newtonian flow, which was indeed often observed in experiments $[26,27,28]$. However, early transition was also reported for certain experimental conditions $[29,30]$. This complex dependence on polymers suggests the coexistence of more than one transition pathways. Recent experiments by Samanta et al. [31] showed that as the polymer concentra- 
tion increases, the origin of the instability driving the transition changes from inertia - the same transition mechanism as Newtonian flow which is only delayed by polymers - to a combination of inertia and elasticity. The latter socalled "elasto-inertial turbulence (EIT)" can appear at Re much lower than $\operatorname{Re}_{\text {crit }} \approx 2100$ of the Newtonian pipe flow and shows distinct flow structures from the coherent structures in inertia-driven turbulence (IDT) [31, 32, 33].

In Newtonian channel flow, the transition to turbulence bypasses the Tollmien-Schlichting (T-S) wave, which does not appear until Re $\approx 5600$ [34], and is able to occur at a much lower $\mathrm{Re}_{\text {crit }} \approx 1000$ [35] with a higher turbulence growth rate $[36,37]$. The process relies on various modes of streak instability where disturbances on streamwise velocity streaks lead to the roll-up and lift-up of vortices, which generates three-dimensional turbulence through a so-called "breakdown" process [38, 39, 40, 41, 42]. Typical practice for studying the bypass transition in DNS is to superpose certain predefined disturbances on a two-dimensional base flow and track the transient evolution of flow structures. For viscoelastic fluids, earlier work largely focused on the linear stability of the base flow [43, 44, 45, 46] whereas the bypass transition, either driven by inertia alone (leading to classical turbulence) or a combination of inertia and elasticity (which leads to EIT), is likely a nonlinear process. Samanta et al. [31] pointed towards a scenario in which these two pathways coexist in parallel. Although the latter, i.e., the laminar-EIT transition, is an emerging topic of interest, understanding of the more classical laminar-IDT pathway is still rather limited in the case of viscoelastic fluid flows. The only nonlinear DNS study so far, to our best knowledge, was reported by Agarwal et al. [47], where the development of a localized 


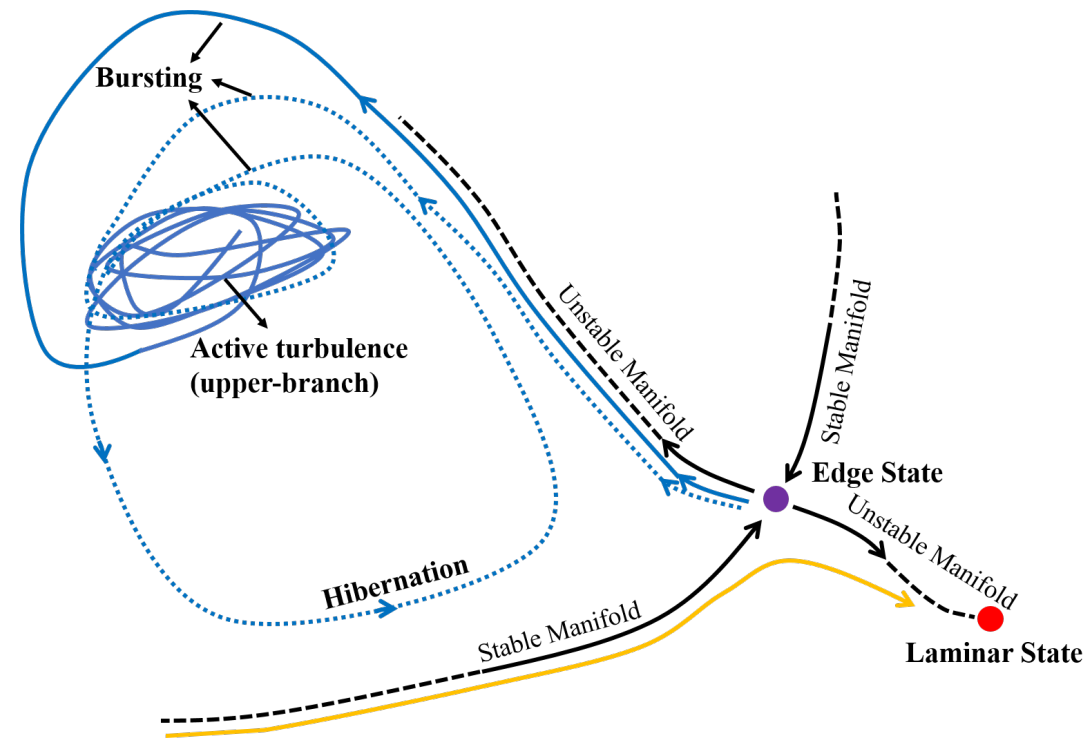

Figure 1: Schematic of the state space of invariant and transient solutions to the NavierStokes equation (based on current understanding). Blue solid line represents the shooting trajectories of this study. The overshoot magnitude (during moments marked with "bursting") is lower when the initial state is more distant from the edge state. Within the turbulent basin, intermittent excursions towards the laminar-turbulent boundary ("hibernation") are pivoted back by the edge state dynamics.

perturbation was tracked and polymers were found to suppress the turbulence growth rate and prolong the transition process. In this study, we aim to further the understanding of the laminar-IDT transition by following the solution objects directly responsible for the process and quantitatively analyze the contributes of inertial, viscous, and elastic forces on the turbulence development.

From a dynamical system perspective, at least at Re not too far above its critical magnitude for the $\mathrm{L}-\mathrm{T}$ transition $\mathrm{Re}_{\text {crit }}$, there are two basins of attraction in the solution state space of the Navier-Stokes equation (fig. 1). 
The laminar state is a linearly stable steady state whereas turbulence can be viewed as a chaotic attractor formed around a number of invariant saddle points $[48,49]$. In the context of L-T transition, one saddle point of particular interest is the so-called edge state (ES) [50,51], whose stable manifold forms the boundary between the basins. Dynamical trajectories initiated from different sides of the boundary head towards opposite directions, following the unstable manifold of the ES. To trigger turbulence from the laminar state, the disturbance must be large enough to overpass the L-T boundary. The importance of the ES in governing the L-T transition was clearly illustrated in a recent study of the asymptotic boundary layer flow, where the transient development of turbulence triggered by random noises was found to be mediated by the ES together with its stable and unstable manifolds [52].

With polymers continuously suppressing turbulence and pushing it into weaker forms, the ES is of particular interest to DR research since it is the weakest or most marginal form of turbulence. Numerical solutions of the ES in viscoelastic flow were only computed recently $[53,54]$. Flow structures of these solutions are strongly reminiscent of those of MDR, which feature smooth velocity streaks, weak vortices, and a kinematics dominated by shear motion [54]. In these states, polymer molecules are not sufficiently stretched and the polymer stress is limited; consequently, flow statistics of the ES is confirmed to be insensitive to polymer effects. The existence of weak but self-sustaining turbulent states unaltered by drag-reducing polymers not only explains the existence of MDR - i.e., why polymers are not able to completely quench turbulence to the laminar state, it also offers a perfect explanation to the universality of MDR: these weak turbulence states are intrinsically 
Newtonian whose flow statistics are not affected by polymers. Dynamic convergence towards the ES with increasing polymer elasticity was demonstrated in DNS solutions of minimal flow units (MFUs), where intermittent quiescent phases similar to the ES (and thus MDR) were identified in the trajectories and phenomenologically termed "hibernating" turbulence [55, 23, 56]. As sketched in fig. 1 with a green line, a turbulent dynamical trajectory spends most of its time sampling the region near the upper-branch (UB) traveling wave solutions, which forms the center of strong "active" turbulence. Hibernating turbulence occurs when excursions are made towards the low-branch (LB) solutions [57], which were know to form the ES [58]. Transition to hibernating turbulence is rare in the Newtonian flow, but polymers are able to suppress active turbulence and increase the frequency of hibernation, leading to a flow that increasingly resembles MDR. Taking an ergodic view of turbulent dynamics, this intermittent transition between active and hibernating intervals in an MFU should be mapped to spatial intermittency between active and hibernating patches in larger domains, which was confirmed in recent experimental and simulation studies $[59,60]$.

The framework constructed so far is solely based on the dynamics around TW solutions, steady states in moving reference frames, with many transient components overlooked. In particular, the so-called "bursting" events - intermittent but violent eruptions of turbulent activities in near wall flow [61] - reflect the importance of non-steady-state dynamics in the turbulent selfsustaining cycle [62]. Non-trivial interaction between turbulent bursting and polymer additives was observed in experiments [63], but its nature has not been explored. Interest into bursting events became rekindled as our recent 
study proposed the suppression of bursting by polymers as a key step in the transformation of turbulent regeneration dynamics at the LDR-HDR transition [25]. Our results (shown below) will reveal that flow structures and dynamics following the unstable manifold of the ES closely resemble typical descriptions of bursting events, offering the first opportunity of studying the polymer effects thereon. Also missing from the current framework is EIT which is an additional solution object that shows up at higher Wi and, as we discuss later, can co-exist with known solutions in fig. 1. The positioning of this solution in the state space and its dynamical relationship with existing ones are unclear as of yet, which is a subject of much future interest. Meanwhile, despite addressing the existence and universality of MDR, the quantitative origin of the Virk asymptote remains unsolved. Although the ES mean velocity follows the Virk asymptote in one particular case [53], departure from the asymptote was observed with changing Re and domain size [54]. Filling in the aforementioned gaps in the framework is necessary for a full understanding of the phenomenon.

It becomes clear now that understanding the dynamics surrounding the ES and its unstable manifold is important for answering many of these questions, especially the bypass transition via streak breakdown and polymerbursting interactions. In this study, we take a non-traditional approach by focusing not on the turbulence at the statistically-converged stage (SCS; i.e., turbulent basin) but in the regime near the ES, and focusing not on the time-averaged statistics but on the transient dynamics. DNS shooting trajectories are initiated from the ES and their dynamics leading to the turbulent basin are tracked (see the blue line in fig. 1). (We use the term 
"shooting" to indicate that the time-integration DNS trajectories are designed to shoot from specific initial states and in specific directions, for a targeted exploration of certain regions in the state space. This usage is similar in spirit as the reference of "shooting" in the transition path sampling method widely used in molecular simulation [64]. This is not to be confused with the shooting method for numerically solving boundary value problems.) Traditional approaches for bypass simulation, as reviewed above, rely on arbitrary perturbation of a base flow. The choice of initial disturbance inevitably affects the observations, which complicates any comparison between studies. Our shooting approach focuses on the turbulence growth from the ES, which shows well-defined streaky flow patterns typically observed before breakdown, and along its unstable manifold. This allows direct access to the solution objects pivotal to various bypass trajectories and offers a benchmark for understanding the transition dynamics. Similarly, bursting dynamics is nearly intractable in standard DNS thanks to the chaotic nature of turbulence: no two bursting events are the same and separating them from the rest of the dynamical cycle is nearly impossible. This study allows the direct comparison of bursting events between Newtonian and viscoelastic flows along the same trajectories, offering the first such opportunity to investigate the polymer effects on bursting dynamics.

\section{Methodology: formulation and simulation details}

We focus on flow in the plane Poiseuille geometry (fig. 2) driven by a constant mean pressure gradient. The $\mathrm{x}, \mathrm{y}$ and $\mathrm{z}$ coordinates are the streamwise, wall-normal and spanwise directions respectively. Lengths are scaled 


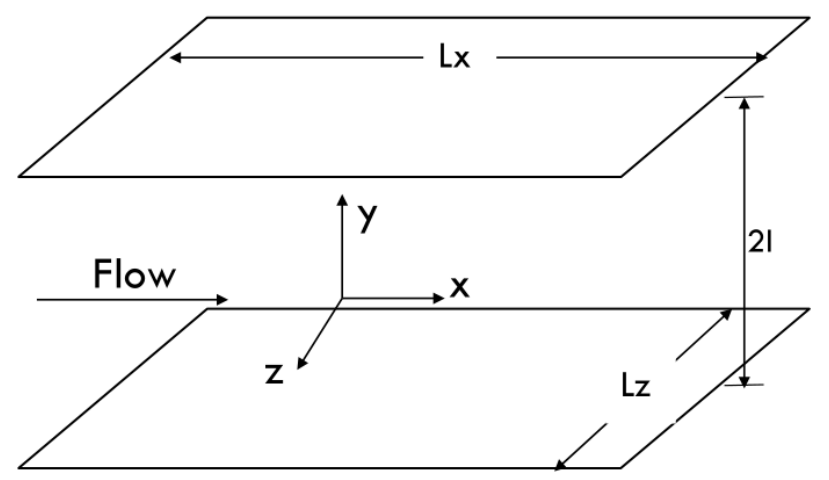

Figure 2: The plane Poiseuille geometry.

with the half-channel height $l$, velocities are scaled with Newtonian laminar center-line velocity $U_{\mathrm{CL}}$, time is scaled with $l / U_{\mathrm{CL}}$, and pressure is scaled with $\rho U_{\mathrm{CL}}^{2}$. The no-slip boundary condition is applied at the walls $(y= \pm l)$ and periodic boundary conditions are adopted in $\mathrm{x}$ and $\mathrm{z}$ directions, in which the periods are denoted as $L_{x}$ and $L_{z}$.

The conservation equations of momentum and mass are:

$$
\begin{gathered}
\frac{\partial \boldsymbol{v}}{\partial t}+\boldsymbol{v} \cdot \boldsymbol{\nabla} \boldsymbol{v}=-\boldsymbol{\nabla} p+\frac{\beta}{\operatorname{Re}} \nabla^{2} \boldsymbol{v}+\frac{2(1-\beta)}{\operatorname{ReWi}}\left(\boldsymbol{\nabla} \cdot \boldsymbol{\tau}_{p}\right) \\
\boldsymbol{\nabla} \cdot \boldsymbol{v}=0 .
\end{gathered}
$$

Here, the Reynolds number is defined as $\operatorname{Re} \equiv \rho U_{\mathrm{CL}} l / \eta$, where $\rho$ is the total density of the fluid; $\eta \equiv \eta_{s}+\eta_{p}$ is the total zero-shear-rate viscosity of the fluid and $s$ and $p$ represent solvent and polymer contributions respectively. The Weissenberg number is defined as $\mathrm{Wi} \equiv 2 \lambda U_{\mathrm{CL}} / l$, the product of the polymer relaxation time $\lambda$ and the mean wall shear rate. The viscosity ratio $\beta \equiv \eta_{s} /\left(\eta_{s}+\eta_{p}\right)$ is the ratio of the solvent viscosity to the total zeroshear-rate viscosity, and $1-\beta$ is approximately proportional to the polymer 
concentration in dilute polymer solutions. We can use $\rho, \eta$ and the mean wall shear stress $\tau_{w}$ to define turbulent inner scales, which are more appropriate in the near-wall region [15]. Therein, velocity is scaled with the friction velocity $u_{\tau} \equiv \sqrt{\tau_{w} / \rho}$, and the viscous length scale $\delta_{v} \equiv \eta / \rho u_{\tau}$. Then the friction Reynolds number is defined as $\operatorname{Re}_{\tau} \equiv \rho u_{\tau} l / \eta$ and the eddy turnover time is $l / u_{\tau}$. Quantities nondimensionalized with these time-averaged inner scales are marked with a superscript "+". Because of the constant mean wall shear stress, $\operatorname{Re}_{\tau}=\sqrt{2 \operatorname{Re}}$ and $l / u_{\tau}=\sqrt{\operatorname{Re} / 2}$ (the latter measured in outer time units - TUs $\left.-l / U_{\mathrm{CL}}\right)$ are both constant. Following earlier studies $[55,23,53,54]$, for flow fields and statistics near one of the walls at a given moment, it is more relevant to use the instantaneous wall shear stress of that wall, $\tau_{w}^{*}$, to define the inner scales; quantities scaled in this way are marked with a superscript "*".

In eq. (2), the last term on the right-hand side contains the polymer stress tensor $\boldsymbol{\tau}_{p}$, which is obtained from the FENE-P constitutive equation (finitely extensible nonlinear elastic dumbbell model with the Peterlin approximation) [65]:

$$
\begin{gathered}
\frac{\boldsymbol{\alpha}}{1-\frac{\operatorname{tr}(\boldsymbol{\alpha})}{b}}+\frac{\mathrm{Wi}}{2}\left(\frac{\partial \boldsymbol{\alpha}}{\partial t}+\boldsymbol{v} \cdot \boldsymbol{\nabla} \boldsymbol{\alpha}-\boldsymbol{\alpha} \cdot \boldsymbol{\nabla} \boldsymbol{v}-(\boldsymbol{\alpha} \cdot \boldsymbol{\nabla} \boldsymbol{v})^{T}\right)=\left(\frac{b}{b+2}\right) \boldsymbol{\delta} \\
\boldsymbol{\tau}_{p}=\frac{b+5}{b}\left[\frac{\boldsymbol{\alpha}}{1-\frac{\operatorname{tr}(\boldsymbol{\alpha})}{b}}-\left(\frac{b}{b+2}\right) \boldsymbol{\delta}\right] .
\end{gathered}
$$

Here $\boldsymbol{\delta}$ is the Kronecker delta tensor. With $\boldsymbol{Q}$ denoting the end-to-end vector of polymer molecules, the conformation tensor is defined as $\boldsymbol{\alpha} \equiv\langle\boldsymbol{Q Q}\rangle(\langle\cdot\rangle$ represents the ensemble average). The polymer extension is limited by an upper limit $b$ : $\max (\operatorname{tr}(\boldsymbol{\alpha}))<b$. In total, the system is specified by four parameters Re, Wi, $\beta$ and $b$. 
All simulations reported in this study are performed at a moderate $\mathrm{Re}=$ $3600\left(\operatorname{Re}_{\tau}=84.85\right)$. (We consider $\mathrm{Re}$ to be low when it is well below $\mathrm{Re}_{\text {crit }}$ for the L-T transition and inertia is not sufficient to drive instability; highRe refers to the regime where turbulence is fully developed. In this study, our Re is only slightly higher than $\mathrm{Re}_{\text {crit }}$, which is the reason for the word "moderate".) Although most experiments are performed at much higher Re, evidence is abundant that qualitative transitions in viscoelastic turbulence can all be observed in the regime close to the L-T transition $[1,14,4]$. This includes the onset of DR, LDR-HDR transition, convergence to MDR, as well as the direct laminar-MDR transition. Meanwhile, $\operatorname{Re}_{\tau}=84.85$ is too low for the complete separation between turbulent inner and outer layers. Therefore, it would be naive to expect the turbulent dynamics and structures to preserve all features of higher Re. Dynamics at the near-transition regime in a way is a toy model for realistic experimental systems: it is a miniaturized version which includes the key components giving rise to these transitions, but many detailed aspects of the dynamics are left out. By avoiding the complexity at higher Re, this Re regime is often a good starting point of investigation and is thus sufficient for this study. On the other hand, computational cost is often the main factor limiting the Re reached by simulation. Although DNS of viscoelastic fluids can reach much higher Re, the cost of numerical solutions for the ES are at least two orders of magnitude higher. ES-initiated shooting simulation also requires much higher special and temporal resolutions. Our choice of Re is also a result of practicality. Viscoelastic cases reported in this study are all for the same $\beta=0.97$ and $b=5000$ but cover three different Wi: 28,40 , and 72 . 
The simulation domain used here is $L_{x}^{+} \times L_{z}^{+}=720 \times 230$. In typical DNS studies this would be considered an MFU or at least close to an MFU [66, 14]. We are however hesitant to use the term because the dynamics near the ES shows drastically different and complex dependence on the domain size than turbulence at the SCS. Recall that the notion of MFU roots in the ergodic assumption of turbulence, that the temporal statistics of the dynamics in an MFU are representative of the spatial and ensemble statistics in an extended flow domain. Therefore at the SCS, although the MFU domain average shows stronger fluctuations, its average over time is still a reasonable approximation of that in realistic extended flow domains. Dynamics at the ES are known to be fundamentally different whose domain size dependence is non-trivial and nearly intractable: e.g., a slight change in the domain size can cause the ES to change between quasi-periodic and chaotic fluctuations [54]. This dependence is not an artifact but intrinsically part of the nature of the ES being the marginal state of turbulence: the form and magnitude of the disturbance field to trigger turbulence is inevitably specific to each domain. This dependence does not go away until $L_{x}^{+}$, e.g., reaches $O\left(10^{4}\right)$ wall units, which is much larger than typical extended domains for SCS $\left(O\left(10^{3}\right)\right.$ wall units) and beyond the reach of viscoelastic simulations. At the very large domain limit, the ES solution, at least in the Newtonian case, becomes spatially localized to a weak turbulent spot surrounded by nearly laminar surroundings [67, 68]. The particular domain size is chosen here for the availability of ES solutions in this domain from our previous study [54], on which the current study is based. If we were to repeat the study in an even slightly different domain, the results would not compare quantitatively with the current study. Except 
for the domain-size sensitivity, it is also a consequence of the transient nature of this study - turbulence can only be quantitatively compared in terms of statistics and only at the SCS. A full domain-size dependence investigation is not possible even for the computationally less expensive Newtonian case, because of the significantly higher computational cost for ES than DNS (discussed in [54]) and the lack of representativeness of any single domain (discussed above). Therefore, in this study, the transient results of the dynamics initiated from the ES are only discussed in terms of the qualitative stages of the transition events. This focus is very different from typical DNS studies based on quantitative and statistical results.

The viscoelastic DNS code used is custom developed based on Channelflow, a $\mathrm{C}++$ library for Newtonian DNS [48]. The equation system is integrated in time with a third-order semi-implicit backward-differentiation-Adams-Bashforth scheme [69]. For spatial discretization, a pseudo-spectral and finite-difference hybrid scheme is implemented. The $\boldsymbol{v} \cdot \boldsymbol{\nabla} \boldsymbol{\alpha}$ term in eq. (4) is discretized with a conservative second-order upwind TVD (total variation diminishing) finite difference scheme [70, 71] whereas a Fourier-Chebyshve-Fourier pseudospectral projection is used for all other spatial derivative terms. It is well know that a pure pseudo-spectral scheme for eq. (4), which is purely convective (no diffusion term), would be numerically unstable for high Wi. A common practice is to introduce an artificial diffusion (AD) term $1 /(\mathrm{ScRe}) \nabla^{2} \boldsymbol{\alpha}$ (where Sc is the Schmidt number) to the right-hand side of eq. (4) [72]. The magnitude of the artificial diffusivity is typically chosen at $1 /(\mathrm{ScRe})=$ $O\left(10^{-2}\right)[8,73,74,75]$, although a much lower $1 /(\mathrm{ScRe})=O\left(10^{-4}\right)$ was used in our previous studies $[14,55,23,53,54,25,76]$. Stress diffusion does occur 
in the physical system as a result of the diffusion of polymer molecules themselves [77]. However, the molecular diffusivity is several orders of magnitude lower than the $\mathrm{AD}$ required for numerical stability (which is thus omitted in the physical model of eq. (4)) [78]. Upwind finite difference schemes offer an alternative that can minimize the effects of this unphysical numerical treatment, in which very often local AD applied to selected grid points (where unphysical values of $\operatorname{tr}(\alpha)$ occur $)$, rather than the entire domain, is sufficient to stabilize the simulation [79]. Comparing these two approaches, it was found recently that global AD can suppress turbulent fluctuations driven by polymer elasticity which become important at the very high Wi limit [33]. In this study, in addition to using an upwind TVD scheme for the convection term in eq. (4), an implicit time-stepping method for enforcing the upperboundedness of the polymer conformation tensor (i.e., $\operatorname{tr}(\alpha)<b$ ) is also implemented according to Vaithianathan et al. [78] and Dubief et al. [11]. Numerically stable solutions are obtained without any AD (global or local) in this study.

For regular DNS (turbulent basin) and edge tracking, the numerical resolution remains the same as our previous studies $[14,55,23,53,54]$ : i.e., $\delta_{x}^{+}=8.57, \delta_{z}^{+}=5.11$, and $N_{y}=73$ Gauss-Lobatto grid points [69] in the $y$ direction $\left(\delta_{y^{+}, \min }=0.081\right.$ at the walls and $\delta_{y^{+}, \max }=3.7$ at the channel center). For DNS shooting from the ES, the rapid turbulence growth and strong overshoot of turbulence intensity (see, fig. 4) require much higher resolution: $\delta_{x}^{+}=3.60, \delta_{z}^{+}=1.80$, and $N_{y}=145\left(\delta_{y^{+}, \min }=0.025 \rightarrow \delta_{y^{+}, \max }=2.28\right) . \mathrm{We}$ have tested multiple resolutions and concluded that the ones reported here are required to fully resolve the small-scale flow structures observed during 


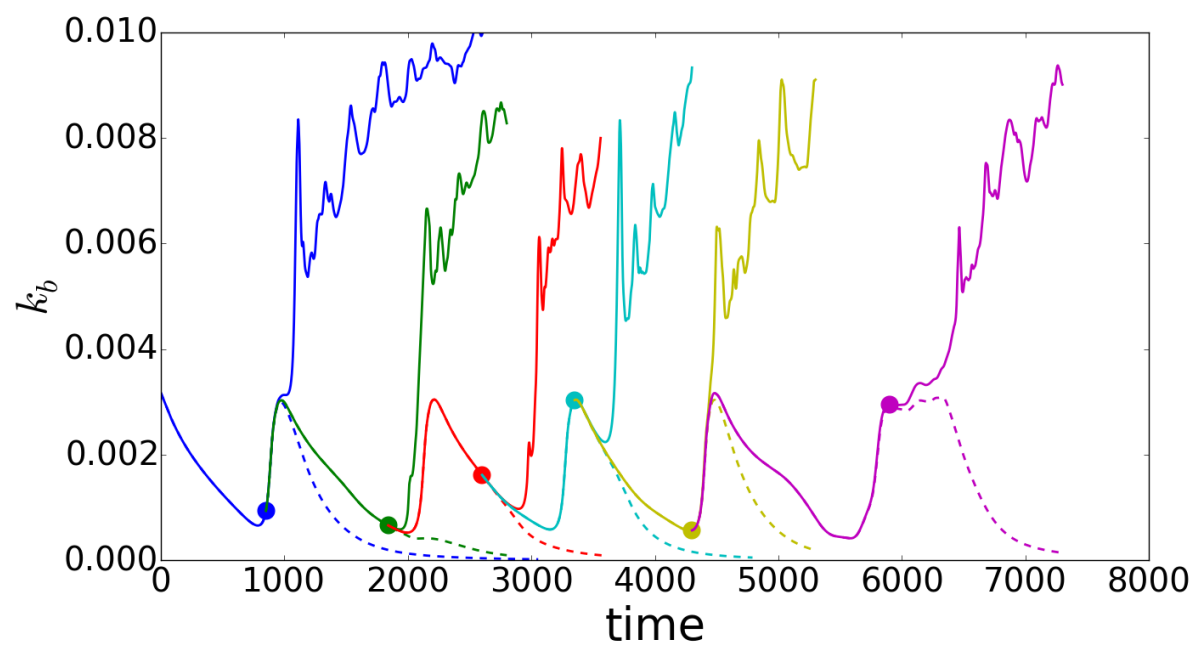

Figure 3: Time series of the bulk-averaged TKE of the twin trajectories used in edge tracking $(\mathrm{Wi}=28)$. Solid and dashed lines are for trajectories on the turbulent and laminar sides, respectively; round dots indicate the points of bisection (see text).

the transition (fig. 8). Our resolution is also higher than that of recent transient DNS studies of the transition [47,80]. The time step size is chosen considering the Courant-Friedrichs-Lewy (CFL) stability condition, which, in shooting runs, is $\delta t=0.004$ and $\delta t=0.005$ for the Newtonian and viscoelastic cases, respectively. The same time step is used for regular DNS (SCS turbulence). For edge tracking, $\delta t=0.02$ is sufficient because of its lower spatial resolution.

The numerical tracking method for computing the ES is shown in fig. 3 in terms of the time series of the bulk-averaged turbulent kinetic energy (TKE) $k_{\mathrm{b}}$. Hereinafter, the subscript "b" denotes bulk- or volume-averaged quantities and

$$
k \equiv \frac{1}{2}\left(v_{x}^{\prime 2}+v_{y}^{\prime 2}+v_{z}^{\prime 2}\right),
$$

where $v_{i}^{\prime} \equiv v_{i}-\left\langle v_{i}\right\rangle$ is the fluctuating velocity component. The method follows 
a pair of DNS trajectories starting from initial states that are infinitesimally close but on different sides of the ES (solid and dashed lines in fig. 3). The twin trajectories move along the ES and thus overlap for a period of time, but eventually diverge, upon which repeated bisection is used to obtain a new pair of infinitesimally-close initial states straddling the ES. The ES is found by connecting the overlapping parts of the twin trajectories. The method has been developed and widely used in the study of Newtonian turbulence and its transition [81, 50, 82, 83] and numerical details for computing the viscoelastic ES used in this study can be found in Xi and Bai [54].

With the current domain size and parameters, the ES itself is a dynamical trajectory showing nearly periodic cycles of relatively strong and weak activities (both are weaker than SCS turbulence; the dynamics may become chaotic in a different domain size - see [54]). The initial state used for all shooting runs (Newtonian and different $\mathrm{Wi}$ ) is taken from a moment of relatively strong activity $\left(\approx 120\right.$ TUs after the peak $k_{\mathrm{b}}$ moment of a period) of the $\mathrm{ES}$ at $\mathrm{Wi}=28$ and from the turbulent side of the twin trajectories so that instabilities can be triggered by numerical error alone. This choice of using the same ES is made so that shooting trajectories of different Wi have the exact same initial condition and can be directly compared. An obvious issue of concern is that other than the $\mathrm{Wi}=28$ case, all shooting runs will have an initial disturbance caused by the mismatch of polymer conformation field to the assigned Wi. Its impact is minimal. First, it has been established in earlier studies that the ES of different Wi (including Newtonian) are nearly identical $[55,54]$. Second, the ES is a saddle point: the shooting trajectory would stay close to the ES for a substantial period of time, during which 
polymers have sufficient time to extend/retract to fit the new $\mathrm{Wi}$, before instabilities start to grow. We have tested the effects of this mismatch by comparing, e.g., the shooting trajectory of $\mathrm{Wi}=40$ using the $\mathrm{Wi}=40 \mathrm{ES}$ as the initial state, with the one presented in this paper (shooting at $\mathrm{Wi}=40$ using the $\mathrm{Wi}=28 \mathrm{ES}$ as the initial state), and found no meaningful difference that would change any of our conclusions. One downside, however, is that shooting trajectories of different Wi would start with different levels of initial disturbance, which, as illustrated in fig. 1, affects the growth rate and overshoot magnitude of the trajectory. This does not affect the objective of this study which focuses on the qualitative stages of transitions. Regarding the choice of the time moment on the ES for the initial shooting point, it is necessary to choose one not too close to the bottom of the periodic fluctuations (fig. 3); otherwise, any additional disturbance would likely tip the dynamics to the other side of the L-T boundary and result in a trajectory heading towards the laminar state. As long as a moment with sufficient turbulent strength for triggering the turbulence growth is picked, qualitatively similar results can be obtained and our results do not lose any generality.

\section{Results and Discussion}

\subsection{Overview of the transition dynamics}

Time series of the Newtonian and viscoelastic DNS shooting trajectories are shown in fig. 4. (The results from all three Wi are qualitatively the same for the time range of interest here - around the initial overshoot and bursting that lead to the turbulent basin. Here, we present the $\mathrm{Wi}=28$

case as an example without the loss of generality in our observations.) The 

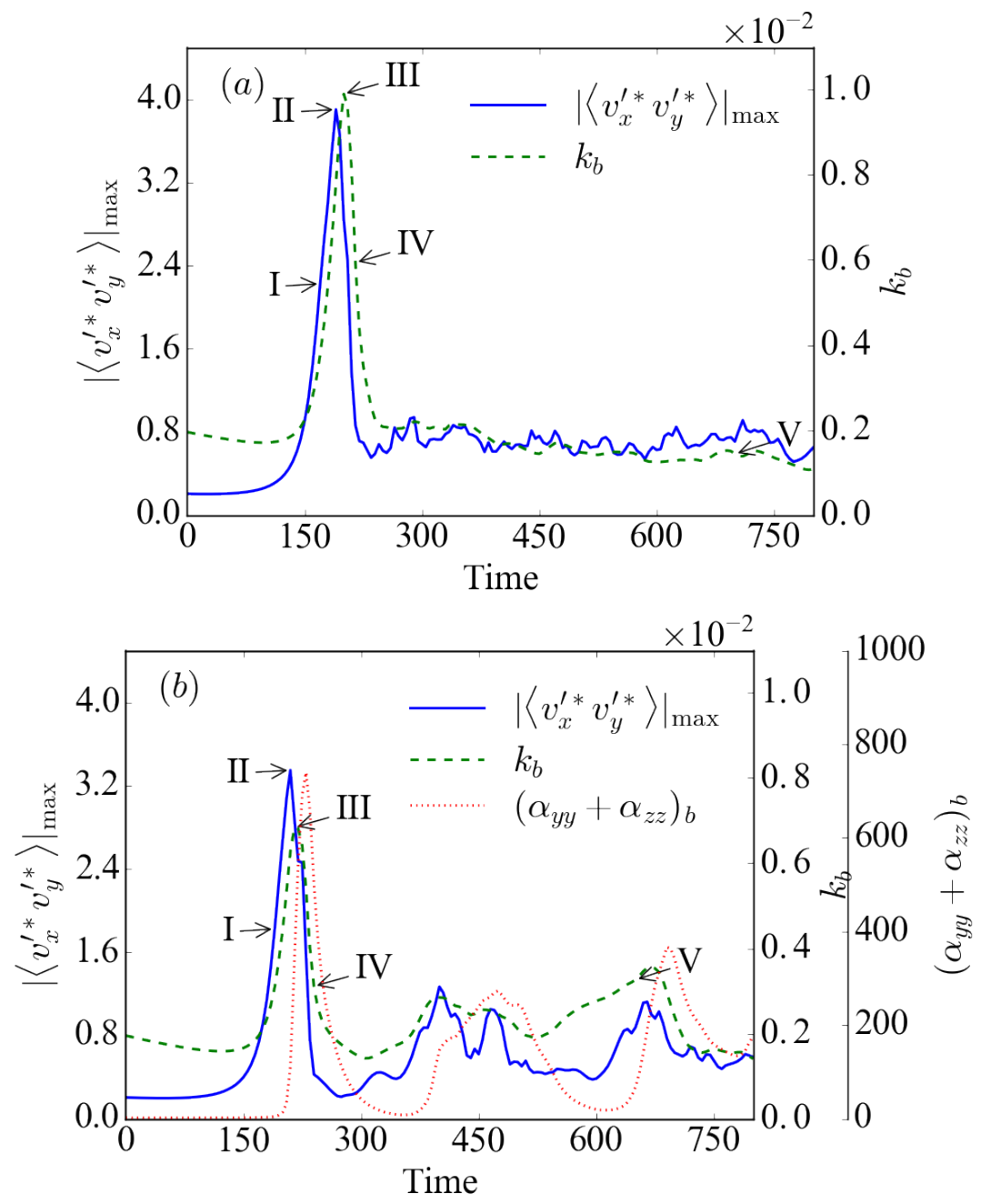

Figure 4: Time series of the shooting trajectories initiated from the ES: (a) Newtonian; (b) $\mathrm{Wi}=28$. The blue solid line (left axis) shows the peak values of the instantaneous RSS profiles; the green dash line (1st right axis) shows the bulk-average TKE; the red dotted line (2nd right axis) shows the bulk average of $\alpha_{y y}+\alpha_{z z}$. 
process of turbulence growth from the ES is qualitatively similar between the Newtonian and viscoelastic cases. The dynamics stays quiescent for a few hundred TUs as the system stays close to the ES. A rapid growth stage follows, starting with a sharp increase in the Reynolds shear stress (RSS). (In fig. 4, the instantaneous $\mathrm{RSS}-v_{x}^{\prime *} v_{y}^{\prime *}$ is averaged in $x$ and $z$; the maximum value of the resulting $y$-dependent profile, denoted by $\left|\left\langle v_{x}^{\prime *} v_{y}^{\prime *}\right\rangle\right|_{\max }$, is plotted against $t$.) In the Newtonian case, the magnitude of $\left|\left\langle v_{x}^{\prime *} v_{y}^{\prime *}\right\rangle\right|_{\max }$ at its peak (marked as moment II) is almost 5 times as large as typical magnitudes of turbulence at the SCS. During the same period, a strong overshoot is also observed in TKE but with a slight phase lag of $\sim 10$ TUs. The peak of TKE is marked as moment III and as we will discuss below, moments II and III have drastically different flow structures. After the overshoots, the RSS quickly drops as the flow enters the SCS where the Newtonian and viscoelastic cases differ significantly. In particular, the viscoelastic case is marked by strong intermittency between periods of quiescent dynamics and those with stronger turbulent intensity, corresponding to hibernating and active turbulence. For example, a pronounced hibernating period can be identified at $480 \lesssim t \lesssim 600$, which is followed by a turbulent overshoot at $t \sim 650$. Fluctuations in the SCS of Newtonian turbulence are more chaotic but contained in a smaller range. Hibernating turbulence can still occur in the Newtonian limit but at a much lower frequency $[55,23]$ : no clear hibernating period is captured in the small time window shown in fig. 4(a).

Similarities between hibernation dynamics and the ES-initiated shooting trajectories can be observed by comparing with a longer time series of viscoelastic turbulence at its SCS (fig. 5). (Note that statistics are taken only 


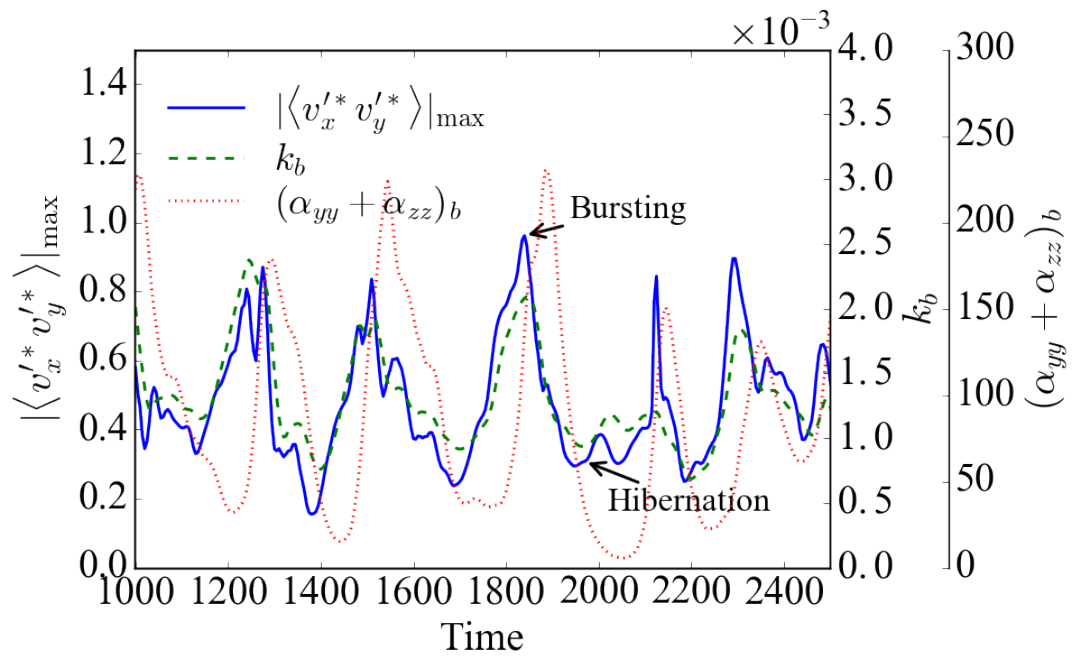

Figure 5: Time series of the turbulent at the SCS at $\mathrm{Wi}=28$ : the blue solid line (left axis) shows the peak values of the instantaneous RSS profiles; the green dash line (right axis) shows the bulk-average TKE $k_{\mathrm{b}}$. Statistics are taken over half of the channel (one side of the center plane). 
over half of the channel because the dynamics near the two walls are not always in sync: mirror average between the two sides will smear the intermittent patterns. Half-channel statistics are also used in fig. 10.) A number of hibernating intervals are identified where the RSS takes a deep dive, e.g., around $t \approx 1400,1750,2000$ and 2200, and each time the flow comes out of hibernation, a strong overshoot is observed. Same as the transient growth in fig. 4, the spikes of TKE appear immediately after those of RSS. This sequence of events is a direct consequence of the TKE production mechanism (discussed below). Overshoots in RSS, TKE, and wall shear stress were also observed immediately after hibernating intervals in previous studies on the topic [55, 23] and even in earlier Newtonian MFU studies before the term hibernating turbulence was coined. For example, Webber et al. [84] noted the intermittent occurrence of the so-called "entropy events" where the TKE drops to very low levels before quickly rising to sharp peaks. The observation here is also consistent with the scenario sketched in fig. 1: the flow intermittently breaks the entrapment in active turbulence (around the UB solutions) and makes visits to the ES; on its return route, it travels along the direction of (but, as discussed below, not necessarily very close to) the unstable manifold of the ES and experiences a strong spike in turbulent activities, before decaying back to active turbulence. Jiménez et al. [62] once argued that the dynamics of turbulent self-sustaining cycles cannot be completely described by invariant solutions (both UB and LB ones): strong and quick "bursting" events, which correspond to spikes observed here and are likely transient in nature, make an essential component. In light of this, the earlier framework of active-hibernating transition $[55,23,4]$ should now be extended to a three- 
stage cycle consisting of the active turbulence (around the UB), hibernation (near the ES), and bursting. Since polymers are known to shorten the time spent at active turbulence and increase the turnover frequency of these intermittent cycles [55, 23], understanding how polymers affect the bursting dynamics will be essential for a full picture of turbulence approaching MDR. In addition, our recent study proposed that the LDR-HDR transition is a reflection of changes in the self-sustaining dynamics: a dominant pathway for vortex regeneration in Newtonian flow relies on bursting for redistributing disturbances and its suppression by polymers was hypothesized as a key element driving the transition [25]. The shooting approach used in this study allows us to avoid the complexity of tracking the intermittent bursting events at the SGS and directly compare the bursting dynamics between Newtonian and viscoelastic cases on an equal footing.

Streamwise velocity in the $x-z$ plane at $y^{+}=24.85$ is shown in figs. 6 and 7 for these two cases. The initial state from the ES (fig. 7(a)) shows one pair of low- and high-speed streaks in the domain. The streaks are straight in the streamwise direction and weak in magnitude: the velocity variation between them is small compared with the range of the color map. For both Newtonian and viscoelastic cases, as instability starts to develop (moment I, fig. 6(a) \& fig. 7(b)), the low-speed streak splits into two. These streaks further intensify as the RSS increases up to its peak magnitude at moment II, where streamwise variation becomes clearly visible. Observations between the Newtonian and viscoelastic cases start to differ at moment III. In the former (fig. 6(c)\&(d)), streak instability quickly breaks down the structure, leading to a domain filled by small-scale and high-intensity veloc- 
(a) $\mathrm{I}: t=170$

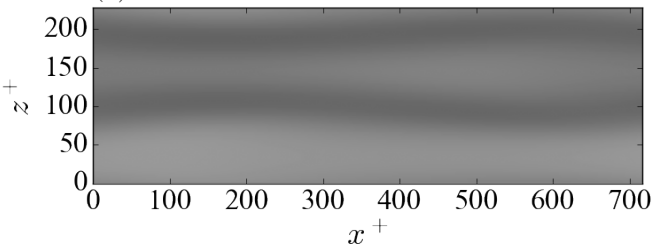

(c) III : $t=200$

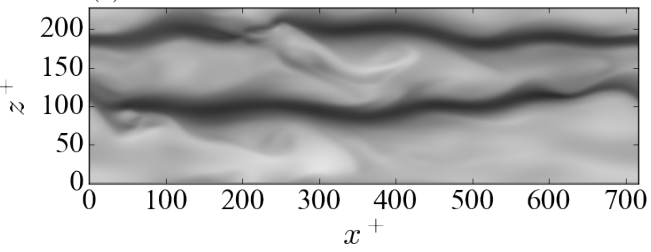

(e) $\mathrm{V}: t=700$

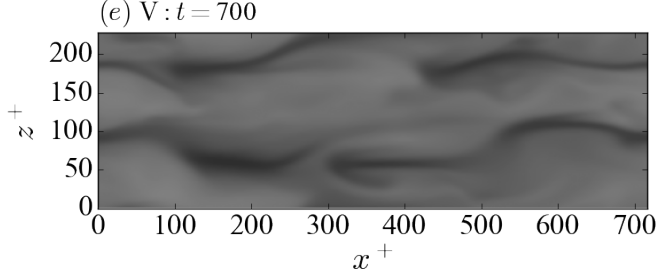

(b) II $: t=190$

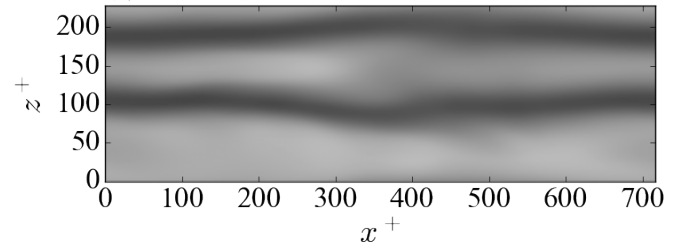

(d) IV $: t=215$

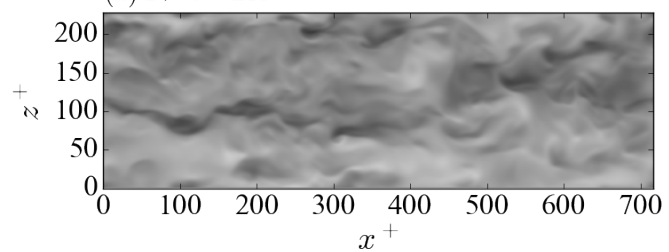

Figure 6: Streamwise velocity distribution at $y^{+}=24.85$ for the Newtonian case: (a) $\sim(\mathrm{e})$ - Moments I V. The initial condition is the same as the viscoelastic case shown in fig. 7(a). The color ranges from black to white for 0 to 1 . 
(a) I.C.

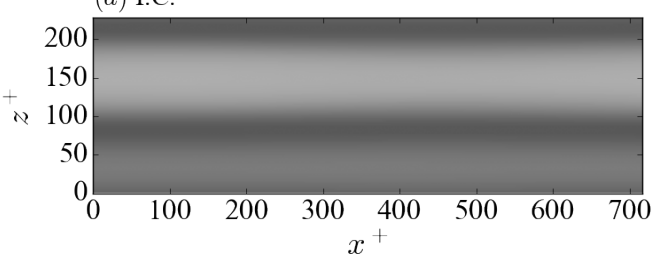

(c) II : $t=210$

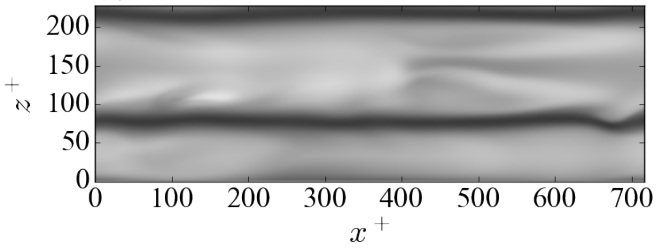

(e) IV $: t=240$

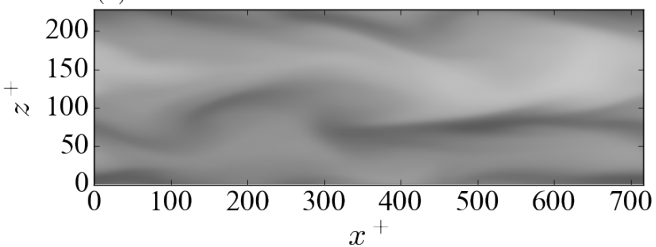

(b) $\mathrm{I}: t=190$

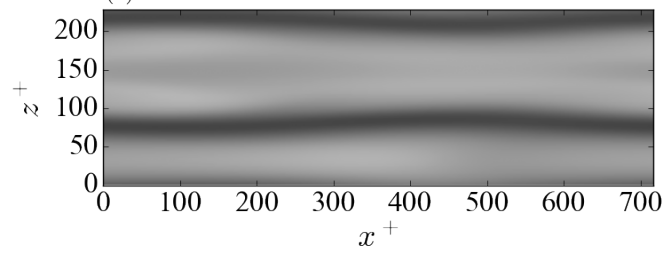

(d) III $: t=215$
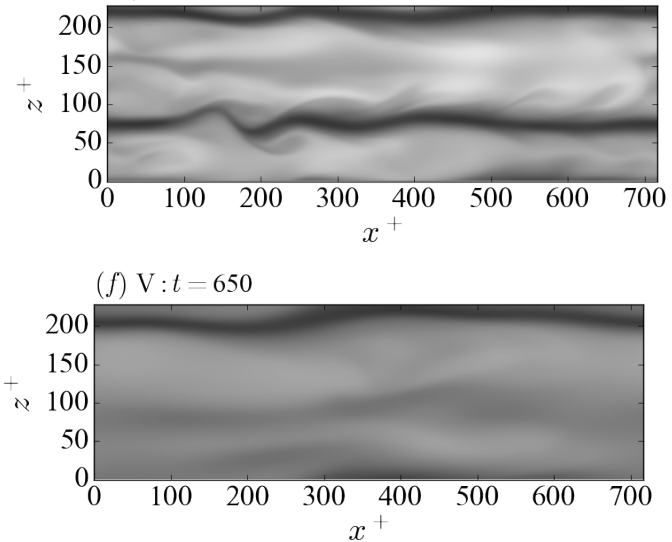

Figure 7: Streamwise velocity distribution at $y^{+}=24.85$ for the $\mathrm{Wi}=28$ case: (a) initial condition; (b) (f) Moments I V . The color ranges from black to white for 0 to 1 . 

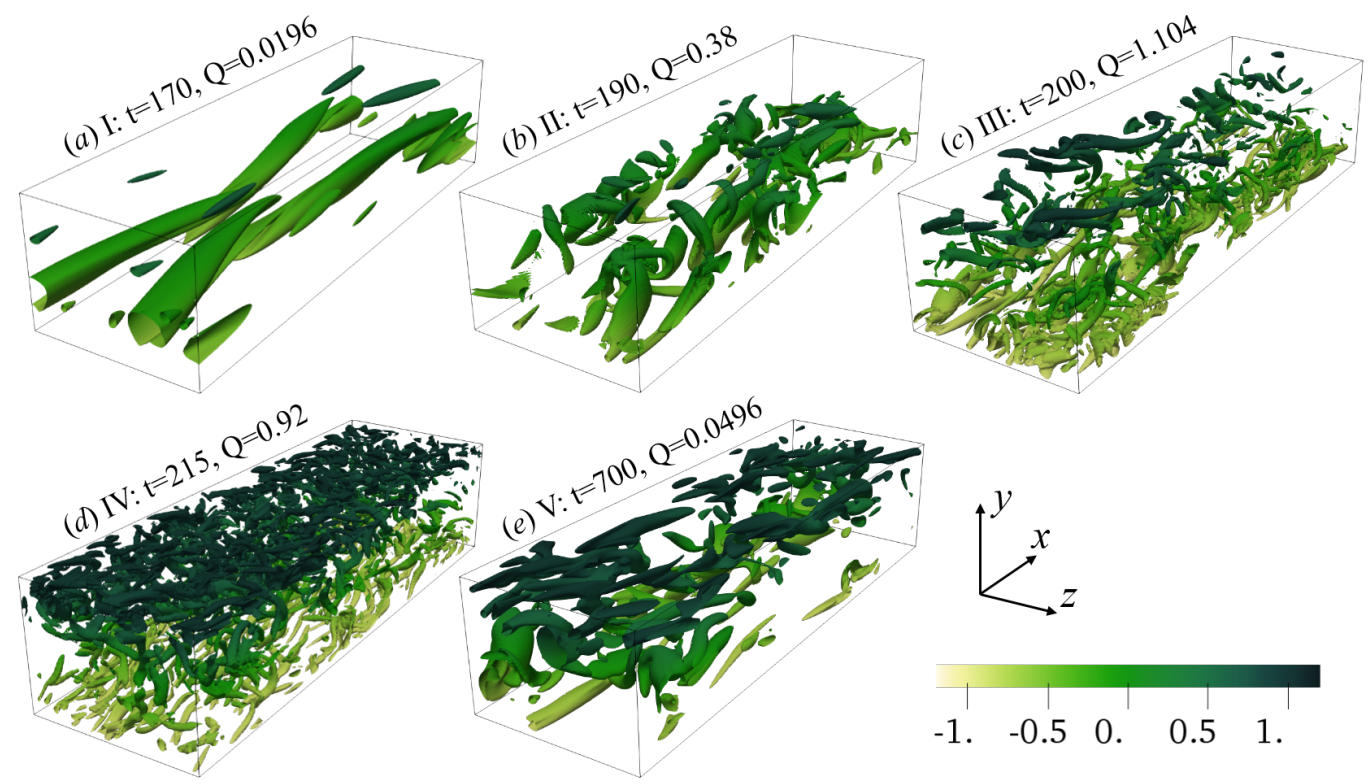

$-1 . \quad-0.5 \quad 0 . \quad 0.5 \quad 1$.

Figure 8: Vortex configuration of the Newtonian case: (a)-(e) are moments I-V. The initial condition is the same as the viscoelastic case shown in fig. 9(a). The isosurface of $Q=Q_{\mathrm{rms}}$ is shown, where $Q_{\mathrm{rms}}$ is the root mean square of the $Q$ field.

ity fluctuations as it leaves the bursting stage. These fluctuations reorganize into streaks with the characteristic spanwise streak spacing of $\sim 100$ wall units [85] as the flow enters the center of the turbulent basin (moment V; fig. 6(e)). By contrast, at high Wi, this breakdown into high-intensity fluctuations is largely avoided and the streaky structure persists through moments III and IV.

Vortex configuration for the same moments is shown in figs. 8 and 9 , where the $Q$-criterion for vortex identification is used [86]. Vortex strength is measured with the scalar field

$$
Q \equiv \frac{1}{2}\left(\|\boldsymbol{\Omega}\|^{2}-\|\boldsymbol{\Gamma}\|^{2}\right)
$$



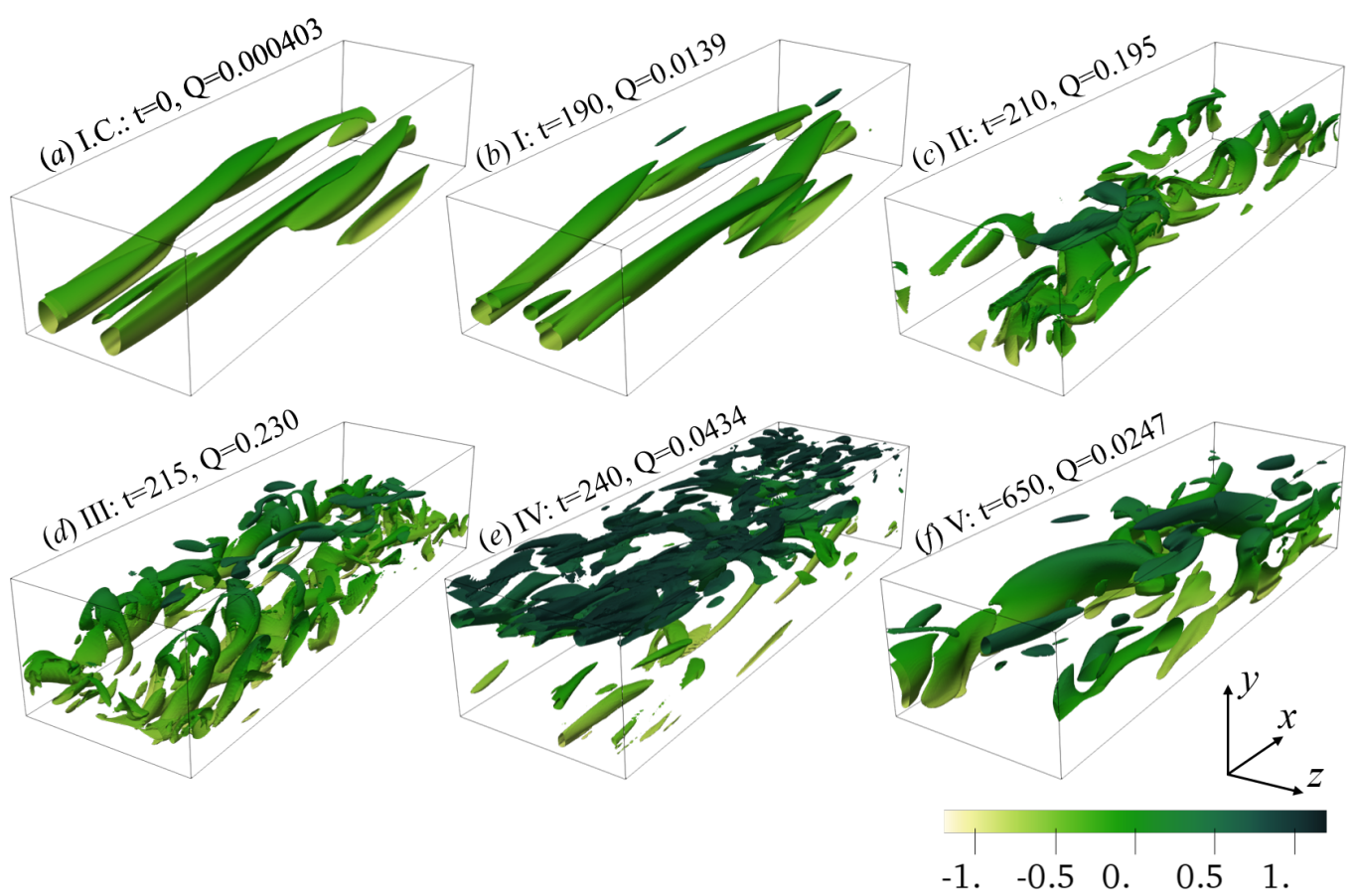

Figure 9: Vortex configuration of the $\mathrm{Wi}=28$ case: (a) initial condition and (b)-(f) are moments I-V. The isosurface of $Q=Q_{\mathrm{rms}}$ is shown, where $Q_{\mathrm{rms}}$ is the root mean square of the $Q$ field. 
where

$$
\boldsymbol{\Omega} \equiv \frac{1}{2}\left(\boldsymbol{\nabla} \boldsymbol{v}-\boldsymbol{\nabla} \boldsymbol{v}^{T}\right)
$$

is the vorticity tensor and

$$
\Gamma \equiv \frac{1}{2}\left(\boldsymbol{\nabla} \boldsymbol{v}+\boldsymbol{\nabla} \boldsymbol{v}^{T}\right)
$$

is the rate of strain tensor; $\|$. $\|$ represents the Frobenius tensor norm. The initial state from the ES shows weak vortices (the root mean square RMS - of $Q$ values in the domain, $Q_{\mathrm{rms}}=2.37 \times 10^{-4}$ ), which are mostly straight along the streamwise direction and localized at one side of the channel. Asymmetry and structural localization is typical of the ES in Poiseuille flow [54]. The growth of instability during the rise of the RSS is similar between the Newtonian and viscoelastic cases, where the streak intensification (figs. 6 and 7) is accompanied by the strengthening of the vortices: $Q_{\mathrm{rms}}$ increases to 0.0196 at moment I and 0.38 at moment II in the Newtonian case. Meanwhile the vortices spread to most of the channel and their shape becomes distorted in all three dimensions, which is also reflected in the streak waviness observed in figs. 6 and 7. For the Newtonian case, the streak breakdown corresponds to an abrupt change in the vortex configuration. Within the 10 TUs between moments II and III, $Q_{\mathrm{rms}}$ jumps from 0.38 to 1.104 and a limited number of relatively large vortices at moment II suddenly explodes into numerous small-scale but high-intensity vortices filling the entire domain. Our finding resonates with the observation by Webber et al. [84] of the temporally intermittent bursting events in an MFU, which they called "entropy events": during these events, TKE is initially contained within very few flow modes as its magnitude quickly rises; then within a very short period of time, TKE is suddenly redistributed to a broad spectrum of scales. 
This again is consistent with the notion that the bursting events come from trajectories approaching and following the unstable manifold of the ES to an overshoot in TKE (fig. 1), which happens after excursions towards the ES (hibernating turbulence). In the viscoelastic case, this sudden breakdown at moment III (fig. 9(d)) is largely avoided and vortices evolve continuously into their configuration at the SCS of turbulence. This, together with the observations in fig. 4 indicates that during the transient development of turbulence (and, likely, during the bursting events as well) polymers do not noticeably change the initial growth of instability, which mainly involves the intensification of streaks and distortion of vortical structures; however, after the RSS has reached its peak, polymers stabilize the primary large-scale vortices from breaking up. By avoiding a strong bursting and its small-scale fluctuations, redistribution of turbulent disturbances is minimized, which is consistent with the hypothesized change in the vortex regeneration mechanism during the LDR-HDR transition by Zhu et al. [25].

State-space trajectories of different solution objects are projected onto the $\left|\left\langle v_{x}^{\prime *} v_{y}^{\prime *}\right\rangle\right|_{\max }-A_{25}^{*}$ plane in fig. $10(\mathrm{a})$, where $A_{25}^{*}$ is the value of

$$
A^{*} \equiv y^{*} \frac{\partial U_{\mathrm{m}}^{*}}{\partial y^{*}}
$$

measured at $y^{*}=25$. Note that eq. (10) comes from taking the differentials of both sides of the log-law relationship of the mean velocity

$$
U_{\mathrm{m}}^{*}=A^{*} \ln y^{*}+B^{*}
$$

and $A^{*}$ defined in eq. (10) is thus interpreted as the local log-law slope. For a well-defined log-law region $A^{*}$ would be nearly constant with varying $y^{*}$, which is why it is also referred to as the indicator function by White et al. 

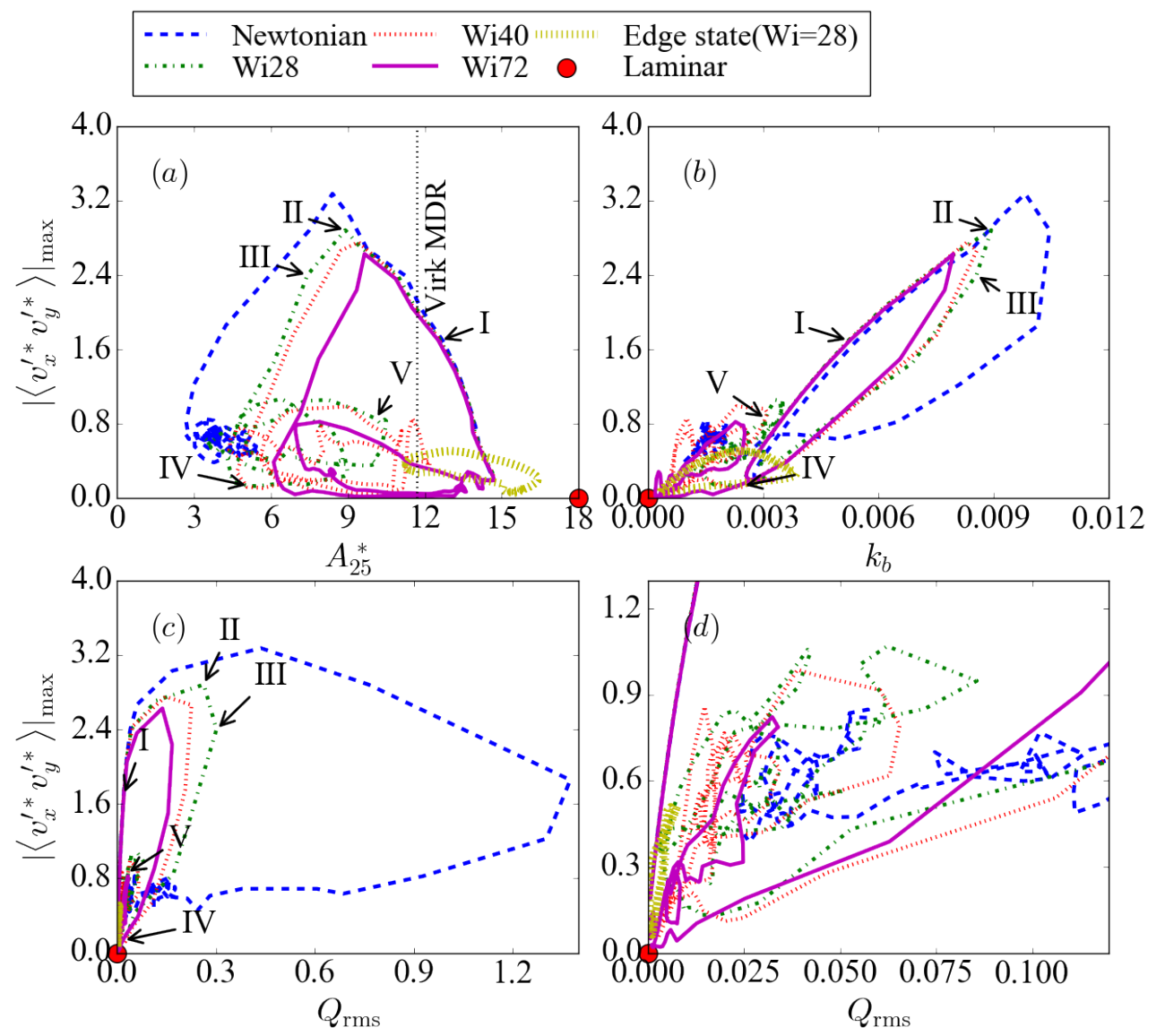

Figure 10: State-space projections of solution trajectories onto different combinations between coordinates: instantaneous RSS profile peak $\left|\left\langle v_{x}^{\prime *} v_{y}^{\prime *}\right\rangle\right|_{\max }$, bulk-average TKE $k_{\mathrm{b}}$, and the RMS of the $Q$ field $Q_{\mathrm{rms}}$. Statistics are taken over half of the channel on the side where the initial ES resides. Panel (d) is a blow-up view of panel (c). Labeling (moments $\mathrm{I} \sim \mathrm{V}$ on the $\mathrm{Wi}=28$ trajectory) is consistent with fig. $4(\mathrm{~b})$. 
[16]. It is used in our projection as a local measurement of the evolution of the mean velocity profile in the near wall layer, which may be compared with the PvK and Virk asymptotes [54]. Transient solutions of Newtonian and three viscoelastic cases, initiated from the same point on the edge state, all move along the ES for a segment before deviation, which is expected for dynamics near a saddle point. After the departure, the RSS quickly rises but the decline in $A_{25}^{*}$ is slow, while different trajectories remain close to one another. Separation between the trajectories only occurs as the RSS reaches its peak, after which the drop of $A_{25}^{*}$ accelerates as the trajectories spiral into regions corresponding to their respective basins of SCS turbulence. Notably, the Virk MDR slope magnitude $A_{\text {Virk }}=11.7$ falls right within the region where trajectories with different Wi (including Newtonian) are overlapping - i.e., before polymer effects on the turbulent dynamics kick in. With increasing $\mathrm{Wi}$, the RSS magnitude at the top of the overshoot decreases (as expected). The turbulent SCS region also shifts in the lower right direction, reflecting the lower friction drag. Compared with the Newtonian trajectory, which concentrates in a small region after reaching the SCS, all viscoelastic trajectories display much higher levels of intermittency and sample broader areas (within about the same time span). Frequent occurrence of hibernating turbulence can be observed in the form of excursions to regions with much weaker turbulence (lower-right corner). For the higher Wi (40 and 72) cases, these excursions sample well into the ES region (viewed from this projection). Similarities between hibernating turbulence and the ES were extensively discussed in previous studies [53, 54], but, here, their dynamical connection also becomes clear. 
The same solution objects are projected to other two-dimensional planes, $\left|\left\langle v_{x}^{\prime *} v_{y}^{\prime *}\right\rangle\right|_{\max }-k_{\mathrm{b}}$ (fig. 10(b)) and $\left|\left\langle v_{x}^{\prime *} v_{y}^{\prime *}\right\rangle\right|_{\max }-Q_{\mathrm{rms}}$ (fig. 10(c)), for a comprehensive view of the dynamics. In both panels, the initial overshoot is displayed as large clockwise loops reaching high magnitudes in all three quantities. Having polymers reduces the overshoot magnitude and the impact is most striking in $Q_{\mathrm{rms}}$, where the peak values reached in all viscoelastic cases tested are one order of magnitude lower than that in the Newtonian case. This is consistent with observations in figs. 8 and 9 that the strong burst of intense turbulence with small-scale fluctuations (moments III \& IV) is clearly circumvented upon the introduction of polymers. After converging to the SCS turbulence, the Newtonian trajectory is confined in a very limited region and shows much lower intermittency than viscoelastic ones. The latter, again, are found to frequently approach the ES during hibernating periods. Subsequent bursting events (as observed in fig. 5) are more clearly shown in these projections (especially in fig. 10(d)) as excursions towards the top-right corner. Time spent within the central region is minimized.

The magnitudes of bursts during these intermittent cycles $\left(\left|\left\langle v_{x}^{\prime *} v_{y}^{\prime *}\right\rangle\right|_{\max }\right.$ up to $\sim 1.0$ and $k_{\mathrm{b}}$ up to $\sim 0.004$ ) are nowhere close to the initial overshoots following the instabilities from the ES. This is because it is very unlikely for the dynamical trajectories to completely reach the ES during the hibernating periods. As illustrated in fig. 1, the overshoot magnitude depends on the proximity of these visits to the ES and its unstable manifold - the strongest overshoot is reached when the trajectory is initiated right on the unstable manifold (which is what the shooting trajectories in this study is designed for). Although during hibernating periods, turbulent structures are remark- 
ably similar to those of the ES [54] and the trajectories seem to repeatedly cut across the ES area in different panels of fig. 10, these visits are not always close to the ES in all dimensions. (Note that projection coordinates used in fig. 10 all measure the instantaneous turbulent fluctuations and local velocity gradient.) Indeed, the distance between hibernation and the ES would be clear in projection quantities involving the mean momentum, such as the bulk-averaged velocity $U_{\mathrm{b}}$. For example, at $\mathrm{Wi}=28, U_{\mathrm{b}}$ reaches up to 0.39 during hibernation whereas its ES value is in the range of 0.61 to 0.62. This is an intrinsic consequence of momentum transport in turbulent flow: increments in the mean momentum during periods of weak turbulence rely on viscous forces. In non-dimensional inertial TUs $\left(l / U_{\mathrm{CL}}\right)$, the time scale for viscous transport is $\operatorname{Re}(O(1000)$ in this study), whereas typical hibernating periods last no more than a couple of hundred TUs [55, 23]. Therefore, new instabilities will emerge and grow before the mean velocity is substantially raised, leading to bursting events much less fierce than the shooting trajectories directly generated from the ES. Other than a dynamical system perspective, this difference can also be understood considering the budgets of TKE and RSS (discussed below) where the production terms of both equations are directly proportional to the mean velocity gradient (eqs. (20) and (24)).

The initial close alignment between trajectories of different Wi indicates that at least during the early stage of turbulence development the impact of polymers is limited. Time series of $\left(\alpha_{y y}+\alpha_{z z}\right)_{\mathrm{b}}$, which measures the polymer extension in the $y-z$ plane, is shown in fig. 4(b). Although polymer extension 
in the three-dimensional space is measured by

$$
\operatorname{tr}(\boldsymbol{\alpha}) \equiv \alpha_{x x}+\alpha_{y y}+\alpha_{z z}
$$

previous studies $[53,23]$ have shown that, even without turbulent motions, substantial polymer stretching is caused by the mean shear of the flow, resulting in a large $\alpha_{x x}$ contribution to $\operatorname{tr}(\alpha)$. Turbulence-induced polymer extension is more clearly observed when only the $\alpha_{y y}$ and $\alpha_{z z}$ components are considered. The magnitude of $\left(\alpha_{y y}+\alpha_{z z}\right)_{\mathrm{b}}$ increases sharply during the overshoot but only as a reaction to the surge in turbulent intensity: the peak of $\left(\alpha_{y y}+\alpha_{z z}\right)_{\mathrm{b}}$ comes $\approx 15$ TUs later than moment III. The same dynamics is observed for the bursting periods following hibernation within SCS turbulence (fig. 5). The implication of this observation is twofold. First, polymer dynamics does not drive the instability. This is not unexpected as the dynamics is constrained to start from the ES whose unstable manifold captures the inertia-driven pathway for instability and turbulence growth. Second, as the trajectory leaves the ES in the state space, it travels through a region with minimal polymer-turbulence interaction before polymer effects become significant. The latter point is consistent with our earlier speculation that in addition to the ES, there is a group of states insensitive to polymer additives (see fig. 1) located in its neighborhood. They collectively form a barrier between the turbulent basin and the laminar state.

On a final note, earlier MFU studies have established that in a small box of $L_{x}^{+} \times L_{z}^{+}=360 \times 230$, the highest Wi where sustained turbulence can still be found is $28[14,56]$. Those studies used an all-pseudo-spectral algorithm with $\mathrm{AD}(\mathrm{Sc}=0.5)$ for $\mathrm{DNS}$, which would quench instabilities driven by elasticity (including EIT). In the current box (twice as long but 
with the same width), inertia-driven turbulence can sustain (within the time range observed) at $\mathrm{Wi}=40$ for certain initial conditions. (Sensitivity to initial conditions is expected at high Wi given the fractal nature of the L$\mathrm{T}$ boundary $[50,51]$.) At the highest Wi reported here $(\mathrm{Wi}=72)$, the trajectory undergoes a few cycles in the basin of IDT and then appears to decay towards laminar flow. New instabilities sometimes grow after the decay period, which leads to EIT. This indicates the co-existence of IDT and EIT within a certain range of Wi. In addition, for all three Wi reported, EIT-like flow structures show up alongside the dominant streak-vortex structures in the basin of IDT (further discussed below). The dynamical landscape in the presence of both solutions is a subject for future inquiry. For the current study, we focus on the trajectory connecting the ES with the IDT basin. It is clear from the comparison of $\left(\alpha_{y y}+\alpha_{z z}\right)_{\mathrm{b}}$ with other time series in figs. 4 and 5, as well as other evidences presented below, that EIT, or elasticitydriven instability in general, is not observed until after the overshoot. At higher Wi, because the IDT basin is no longer an attractor, i.e., the dynamical trajectories are trapped there only for a finite time period before they escape and head elsewhere, the boundary separating IDT from the laminar basin (formed by the ES and its stable manifold - see fig. 1) is what Lebovitz [87] called a "weak" basin boundary. Our results showed that as far as the shooting trajectories are concerned, there is no qualitative difference between the three Wi studied. The highest Wi $=72$ is thus included here to show the Wi effects on the overshoot and bursting process. Lastly, note that this discussion pertains only to the specific domain size used here - changing the domain size will change the Wi for each transition. For example, it is 

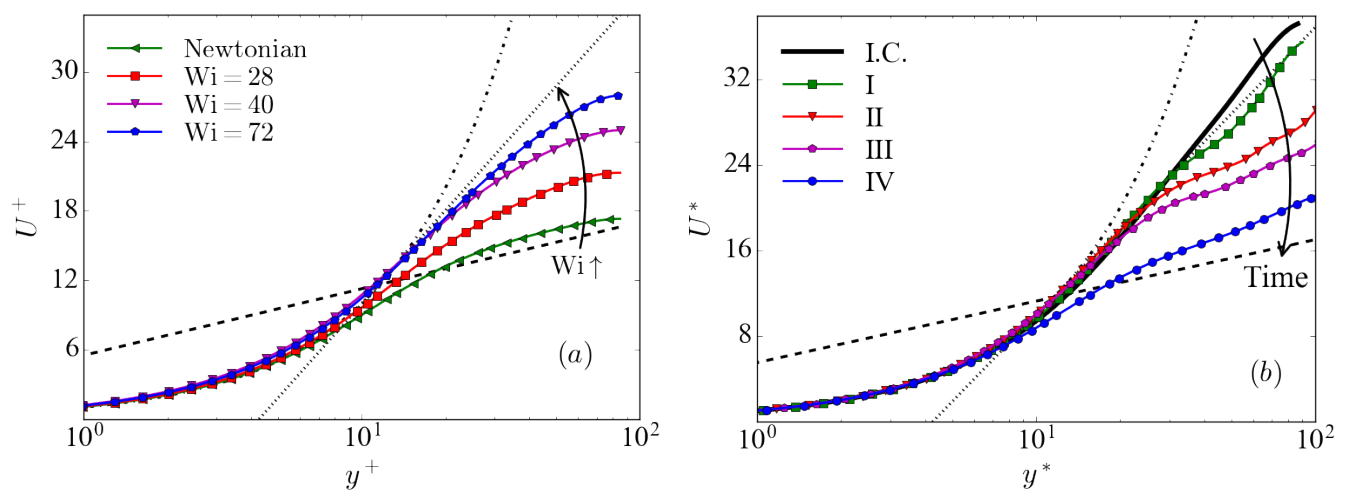

Figure 11: Mean velocity profiles: (a) time-average profiles for Newtonian and viscoelastic cases in the SCS; (b) instantaneous profiles for the initial condition and moments I-IV (as labeled in fig. 4) during the transient development of the $\mathrm{Wi}=28$ shooting trajectory. Reference lines are: (dot-dashed) viscous sublayer, (dashed) PvK log law, and (dotted) Virk asymptote.

clear from earlier studies that the IDT can survive at much higher Wi with increasing domain size $[14,56]$.

\subsection{Mean velocity development and shear stress balance}

The time-averaged mean velocity profile of statistically-converged turbulence (fig. 11(a)) rises from the PvK log law of Newtonian turbulence [88]

$$
U_{\mathrm{m}}^{+}=2.5 y^{+}+5.5
$$

to the Virk asymptote of MDR (eq. (1)) as Wi increases. All profiles overlap with the viscous sublayer asymptote at $y^{+} \lesssim 5$

$$
U_{\mathrm{m}}^{+}=y^{+}
$$

as they should [15]. (At Wi $=72$, IDT does not sustain in the current domain size; therefore the average is taken in a 200-TU window before any sign of its 


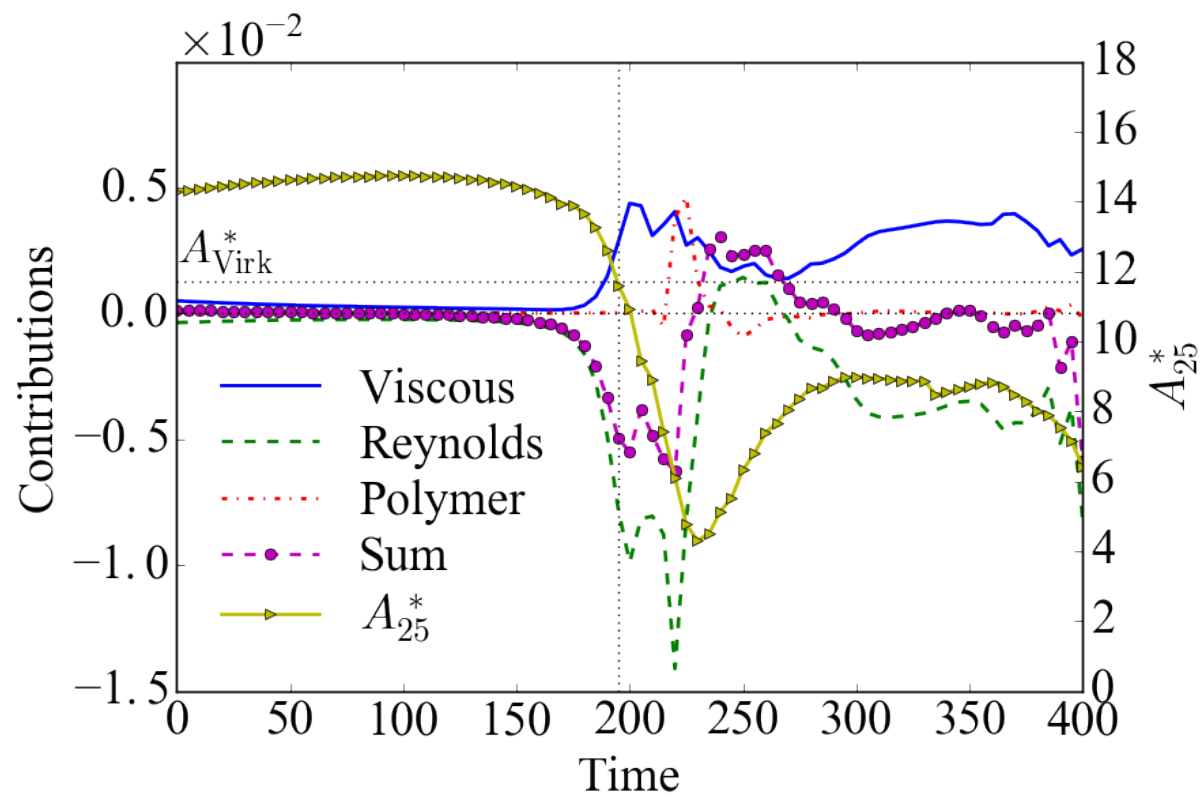

Figure 12: Contributions to the mean velocity gradient development according to eq. (17) (left; "Sum" is the summation of all three contributions) and the log-law slope $A_{25}^{*}$ (right) for $\mathrm{Wi}=28$, calculated at $y^{+}=25$ near the side of the channel where the initial ES resides. Horizontal reference lines are added at the 0 mark of the left axis and the $A_{\text {Virk }}=11.7$ mark of the right axis; the vertical reference line marks the moment when $A_{25}^{*}=A_{\text {Virk }}$. The time axis matches that of fig. 4.

decay.) Instantaneous mean velocity profiles during the transient process of turbulence development are shown in fig. 11(b) for $\mathrm{Wi}=28$. (Since the ES solution is asymmetric $[53,54]$ with respect to the center plane and so is the transient trajectory initiated thereat, hereinafter, instantaneous profiles of the shooting trajectories only show the half channel with stronger turbulent activity.) At the initial stage, the reduction of $U_{\mathrm{m}}$ starts from the channel center (moment I). As time moves forward, this deficit extends into the nearwall layer as the profile drops towards the PvK log law. 
The transient development of mean velocity is governed by the Reynolds equation, which is obtained by taking the $x$-component of eq. (2), applying the Reynolds decomposition to all dependent variables, and taking the ensemble average on both sides. The resulting equation (cast in inner scales where stress and pressure are scaled with $\tau_{\mathrm{w}}$ and time with $\eta / \tau_{\mathrm{w}}$ )

$$
\frac{\partial U_{\mathrm{m}}^{+}}{\partial t^{+}}=\frac{\partial\left\langle\tau_{x y}^{+}\right\rangle}{\partial y^{+}}-\frac{d\left\langle p^{+}\right\rangle}{d x^{+}}
$$

shows that the change of $U_{\mathrm{m}}$ is determined by the streamwise mean pressure gradient and the wall-normal gradient of the mean shear stress

$$
\left\langle\tau_{x y}^{+}\right\rangle \equiv \beta \frac{\partial U_{\mathrm{m}}^{+}}{\partial y^{+}}+\left\langle-v_{x}^{\prime+} v_{y}^{\prime+}\right\rangle+\frac{1-\beta}{\mathrm{Wi}}\left\langle\tau_{\mathrm{p}, x y}\right\rangle .
$$

Recall the definition in eq. (10): $A^{+}$is directly determined by the mean velocity gradient $\partial U_{\mathrm{m}}^{+} / \partial y^{+}$(we use the +-units here instead of the $*$-units to avoid the complexity of changing scaling between different instants; this choice does not qualitatively affect any following discussion), whose evolution is governed by

$$
\frac{\partial}{\partial t^{+}}\left(\frac{\partial U_{\mathrm{m}}^{+}}{\partial y^{+}}\right)=\beta \frac{\partial^{3} U_{\mathrm{m}}^{+}}{\partial\left(y^{+}\right)^{3}}+\frac{\partial^{2}}{\partial\left(y^{+}\right)^{2}}\left\langle-v_{x}^{\prime+} v_{y}^{\prime+}\right\rangle+\left(\frac{1-\beta}{\mathrm{Wi}}\right) \frac{\partial^{2}\left\langle\tau_{\mathrm{p}, x y}\right\rangle}{\partial\left(y^{+}\right)^{2}} .
$$

Equation (17) is obtained by taking the $y^{+}$derivative of both sides of eq. (15) and noting that $d\left\langle p^{+}\right\rangle / d x^{+}$is constant. The three terms on the right-hand side (RHS) describe the contributions from the viscous, Reynolds, and polymer shear stresses, respectively. Time series of these terms are shown in fig. 12 for the transient development period in fig. 4(b). All terms are nearly zero at the beginning when the dynamics is still following the ES. As deviation from the ES starts, the RSS term sharply drops, which drives the downward fall of $A_{25}^{*}$. The polymer shear stress (PSS) term remains close to 
zero until much later (after moment III) and its effect, for the most part, is to raise the mean velocity gradient. This indicates that not only is polymer stress insignificant during the initial overshoot, it also mostly plays a dissipative role in the dynamics of growing turbulence. The viscouse shear stress (VSS) contribution stays positive for the entire process, which also starts well ahead of the PSS. The net effect of all three terms is negative during the entire transition process. Interestingly, at the moment when $A_{25}^{*}=A_{\mathrm{Virk}}$, the driving force to bring down $A_{25}^{*}$ is nearly the largest.

Instantaneous profiles of the VSS, RSS, and PSS - the three contributions to the total shear stress (TSS) defined in eq. (16) - of representative moments are plotted in figs. 13 and 14. The steady-state solution to eq. (15) is

$$
\tau_{x y}^{+}=1-\frac{y^{+}}{\sqrt{2 \mathrm{Re}}}
$$

which is shown as a reference line in all panels. The initial state fig. 14(a), taken from the ES, has a moderate RSS and the VSS is sizeable across the channel; its PSS is negligible. The evolution of the RSS and VSS is qualitatively similar between the Newtonian and viscoelastic cases. As turbulence starts to develop, the RSS quickly increases and reaches its maximum at moment II, which is much higher than the magnitude of the steady-state TSS. (For the Newtonian case, the peak value of moment III is slightly higher in "+"-units, but moment II is still the highest in "*"-units - see fig. 4.) The profile also takes a sharper form, resulting in a large (negative) second-order derivative that quickly suppresses the mean velocity gradient (see eq. (17), fig. 12 and their related discussion). The RSS starts to decay at moment III. At moments IV and V, its shape converges to the typical form in steadystate turbulence which peaks in the buffer layer. The VSS, meanwhile, has 

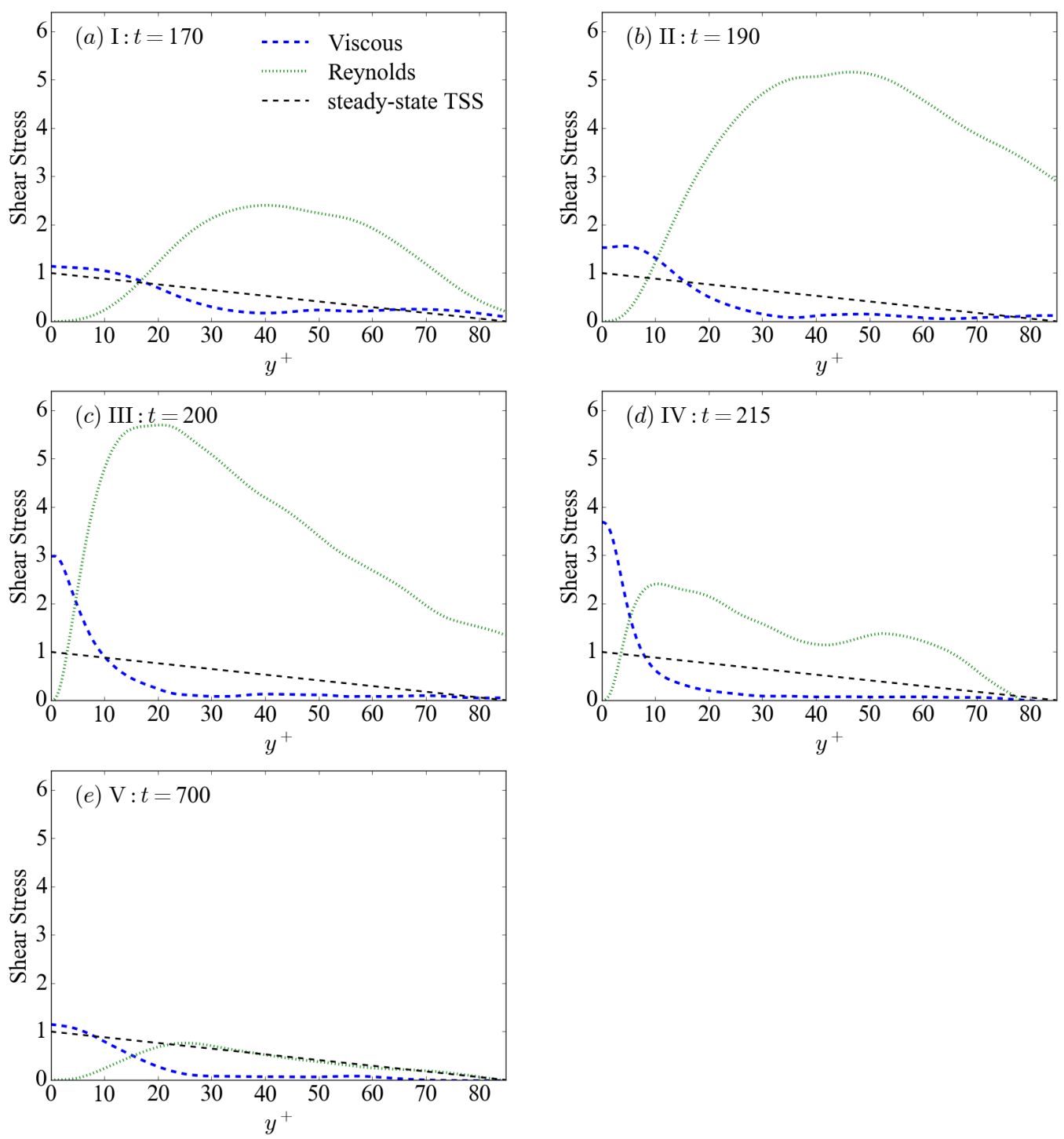

Figure 13: Contributions to the total shear stress (as defined in eq. (16) and in "+" units) in the Newtonian case: $(\mathrm{a}) \sim(\mathrm{e})-$ moments $\mathrm{I} \sim \mathrm{V}$. The initial condition is the same as the viscoelastic case shown in fig. 14(a). Black dashed line is the steady-state TSS (eq. (18)). 

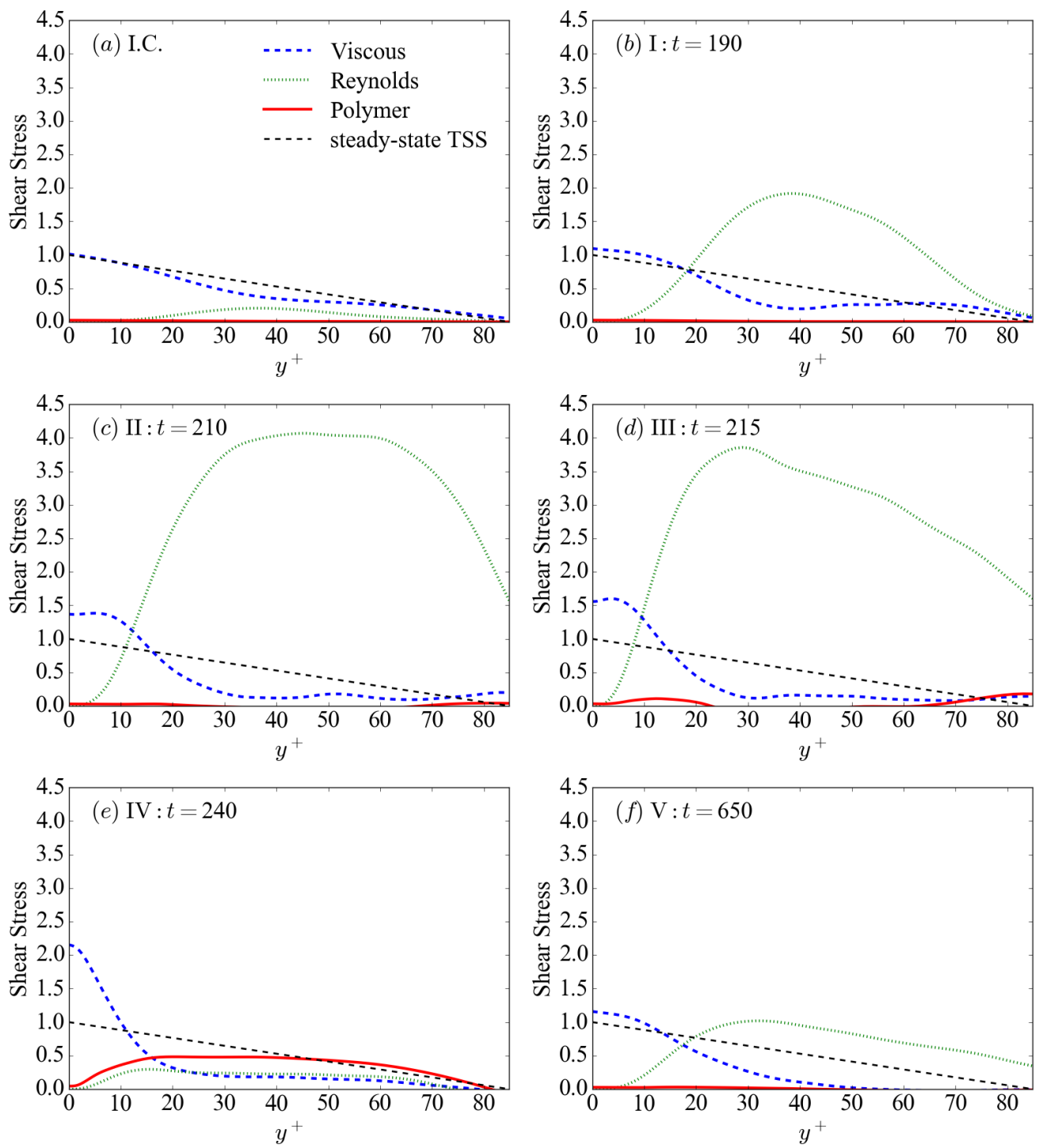

Figure 14: Contributions to the total shear stress (as defined in eq. (16) and in "+" units) in the $\mathrm{Wi}=28$ case: (a) initial condition; (b) $\sim(\mathrm{f})-$ moments $\mathrm{I} \sim \mathrm{V}$. Black dashed line is the steady-state TSS (eq. (18)). 
retreated to the near-wall region only. For the viscoelastic case, the PSS remains small until moment IV, where its magnitude exceeds that of the RSS, whereas at moment V, i.e., within in the SCS, PSS becomes negligibly small once again.

The role of PSS at MDR is an issue often debated. Warholic et al. [89] observed a nearly vanishing magnitude of RSS at MDR. Although the PSS cannot be measured directly in experiments, it was inferred from the shear stress balance (eq. (18)) that the PSS has filled in the deficit left by the RSS. It was thus argued that MDR is a state in which the mechanism of sustaining turbulence in Newtonian flow has been replaced by a polymer-dominated instability. However, other studies (experimental and numerical) showed that the RSS does not necessarily vanish and is not always overpassed by the PSS at MDR [90, 73, 19]. This complexity can be explained now considering the dynamical cycle of the turbulent SCS - hibernation - bursting depicted in fig. 1. Based on fig. 14, large PSS only occurs immediately after the bursting stage. Depending on the relative time spent in different stages, the time-averaged shear stress profiles may or may not show a PSS larger than the RSS.

\subsection{Budgets of the TKE and RSS}

The growth of turbulence as it departs from the ES and the polymer effects thereon are now quantitatively analyzed through the budgets of the TKE and Reynolds stress, which can be derived from the equation of motion (eq. (2)) following the examples of similar equations in Newtonian flow [15]. 
The evolution of TKE is

$$
\frac{\partial k}{\partial t}+\langle\boldsymbol{v}\rangle \cdot \nabla k+\nabla \cdot \boldsymbol{T}^{k}=\mathcal{P}^{k}-\epsilon_{\mathrm{v}}^{k}-\epsilon_{\mathrm{p}}^{k}
$$

where

$$
\mathcal{P}^{k} \equiv-\nabla\langle\boldsymbol{v}\rangle:\left\langle\boldsymbol{v}^{\prime} \boldsymbol{v}^{\prime}\right\rangle=-\left\langle v_{x}^{\prime} v_{y}^{\prime}\right\rangle \frac{d\left\langle v_{x}\right\rangle}{d y}
$$

is the production of TKE (the second equality holds because $d\left\langle v_{x}\right\rangle / d y$ is the only non-zero component of $\boldsymbol{\nabla}\langle\boldsymbol{v}\rangle)$,

$$
\epsilon_{\mathrm{v}}^{k} \equiv \frac{2 \beta}{\operatorname{Re}}\left\langle\Gamma^{\prime}: \Gamma^{\prime}\right\rangle
$$

and

$$
\epsilon_{\mathrm{p}}^{k} \equiv \frac{2(1-\beta)}{\operatorname{ReWi}}\left\langle\boldsymbol{\tau}_{\mathrm{p}}^{\prime}: \boldsymbol{\Gamma}^{\prime}\right\rangle
$$

are the consumption rates of TKE by viscous dissipation and by conversion into elastic energy, respectively $\left(\boldsymbol{\Gamma}^{\prime}\right.$ and $\boldsymbol{\tau}_{\mathrm{p}}^{\prime}$ are the fluctuating components of the rate of strain (eq. (9)) and polymer stress tensors), and $\boldsymbol{T}_{k}$ groups all terms contributing to the flux of $k$ transport, which only moves the TKE spatially but does not convert it to other forms; it therefore will not be further discussed.

The production of the TKE is the product of the RSS $-\left\langle v_{x}^{\prime} v_{y}^{\prime}\right\rangle$ and the mean shear rate $d\left\langle v_{x}\right\rangle / d y$, which explains why the surge in the RSS during the turbulence growth process is followed by a strong spike in the TKE. The evolution of the RSS is governed by

$$
\frac{\partial\left\langle-v_{x}^{\prime} v_{y}^{\prime}\right\rangle}{\partial t}+\langle\boldsymbol{v}\rangle \cdot \nabla\left\langle-v_{x}^{\prime} v_{y}^{\prime}\right\rangle+\nabla \cdot \boldsymbol{T}^{\mathrm{R}}=\mathcal{P}^{\mathrm{R}}+\mathcal{R}^{\mathrm{R}}-\epsilon_{\mathrm{v}}^{\mathrm{R}}-\epsilon_{\mathrm{p}}^{\mathrm{R}}
$$


where

$$
\begin{gathered}
\mathcal{P}^{\mathrm{R}} \equiv\left\langle v_{y}^{\prime} v_{y}^{\prime}\right\rangle \frac{d\left\langle v_{x}\right\rangle}{d y} \\
\mathcal{R}^{\mathrm{R}} \equiv-\left\langle p^{\prime}\left(\frac{\partial v_{x}^{\prime}}{\partial y}+\frac{\partial v_{y}^{\prime}}{\partial x}\right)\right\rangle \\
\epsilon_{\mathrm{v}}^{\mathrm{R}} \equiv-\frac{2 \beta}{\operatorname{Re}} \sum_{w=x, y, z}\left(\left\langle\Gamma_{x w}^{\prime} \frac{\partial v_{y}^{\prime}}{\partial w}\right\rangle+\left\langle\Gamma_{y w}^{\prime} \frac{\partial v_{x}^{\prime}}{\partial w}\right\rangle\right) \\
\epsilon_{\mathrm{p}}^{\mathrm{R}} \equiv-\frac{2(1-\beta)}{\operatorname{ReWi}} \sum_{w=x, y, z}\left(\left\langle\tau_{\mathrm{p}, x w}^{\prime} \frac{\partial v_{y}^{\prime}}{\partial w}\right\rangle+\left\langle\tau_{\mathrm{p}, y w}^{\prime} \frac{\partial v_{x}^{\prime}}{\partial w}\right\rangle\right)
\end{gathered}
$$

are the production, pressure-rate-of-strain, viscous conversion, and elastic conversion terms, respectively; $\boldsymbol{T}^{\mathrm{R}}$ again is the flux of RSS not discussed here. According to eq. (24), at a given mean shear rate, the RSS is generated by wall-normal velocity fluctuations. In the near wall region they are primarily found between streamwise vortices where velocity streaks are formed by the upward lifting and downward flushing fluid motions. For this reason, the rise of the RSS from the ES to moment II is accompanied by the intensification of the streak structures (figs. 7 and 9).

Figures 15 and 16 show the TKE and RSS budgets for typical moments of the Newtonian and $\mathrm{Wi}=28$ cases. The process of the RSS surge (up to moment II) is qualitatively similar between the Newtonian and viscoelastic cases, where the production terms dominate both budgets. During this stage (fig. 15(a) \& (b), fig. 16(a), (b), \& (c)), the RSS production $\mathcal{P}^{\mathrm{R}}$ takes a flatter shape and is larger at around $y^{+}=40$, the location of the primary steaks and vortices at the ES (see fig. 9(a) and [54]), than in the buffer layer; whereas the TKE production $\mathcal{P}^{k}$ peaks in the buffer layer - at $y^{+} \approx 25$. Abrupt changes occur between moments II and III. In the Newtonian case (fig. 15(c)), magnitudes of both $\mathcal{P}^{k}$ and $\mathcal{P}^{\mathrm{R}}$ sharply increase. In addition, $\mathcal{P}^{\mathrm{R}}$ 

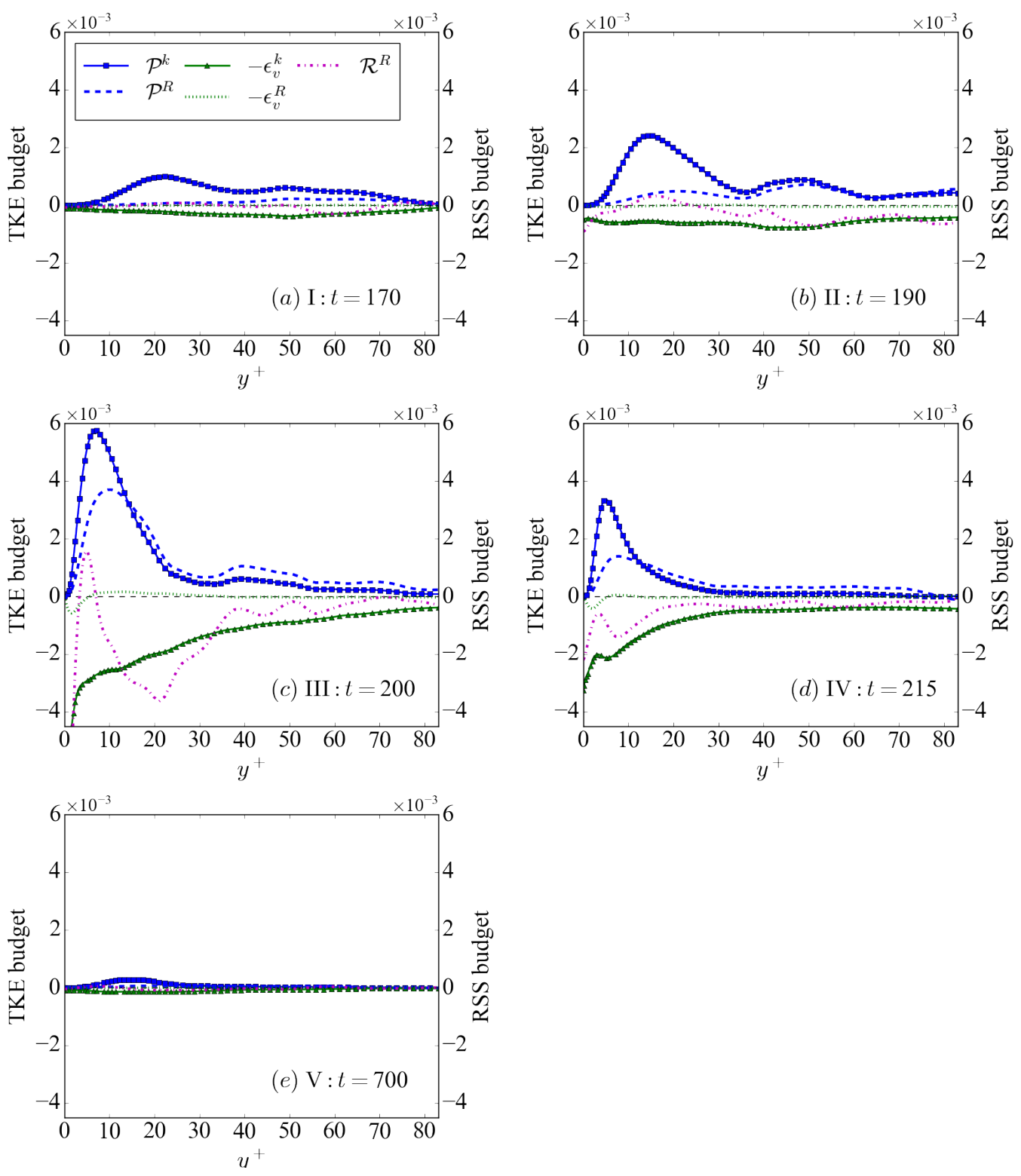

Figure 15: TKE and RSS budgets for the Newtonian case: (a)-(e) are moments I-V. The initial condition is the same as the viscoelastic case shown in fig. 16(a). 

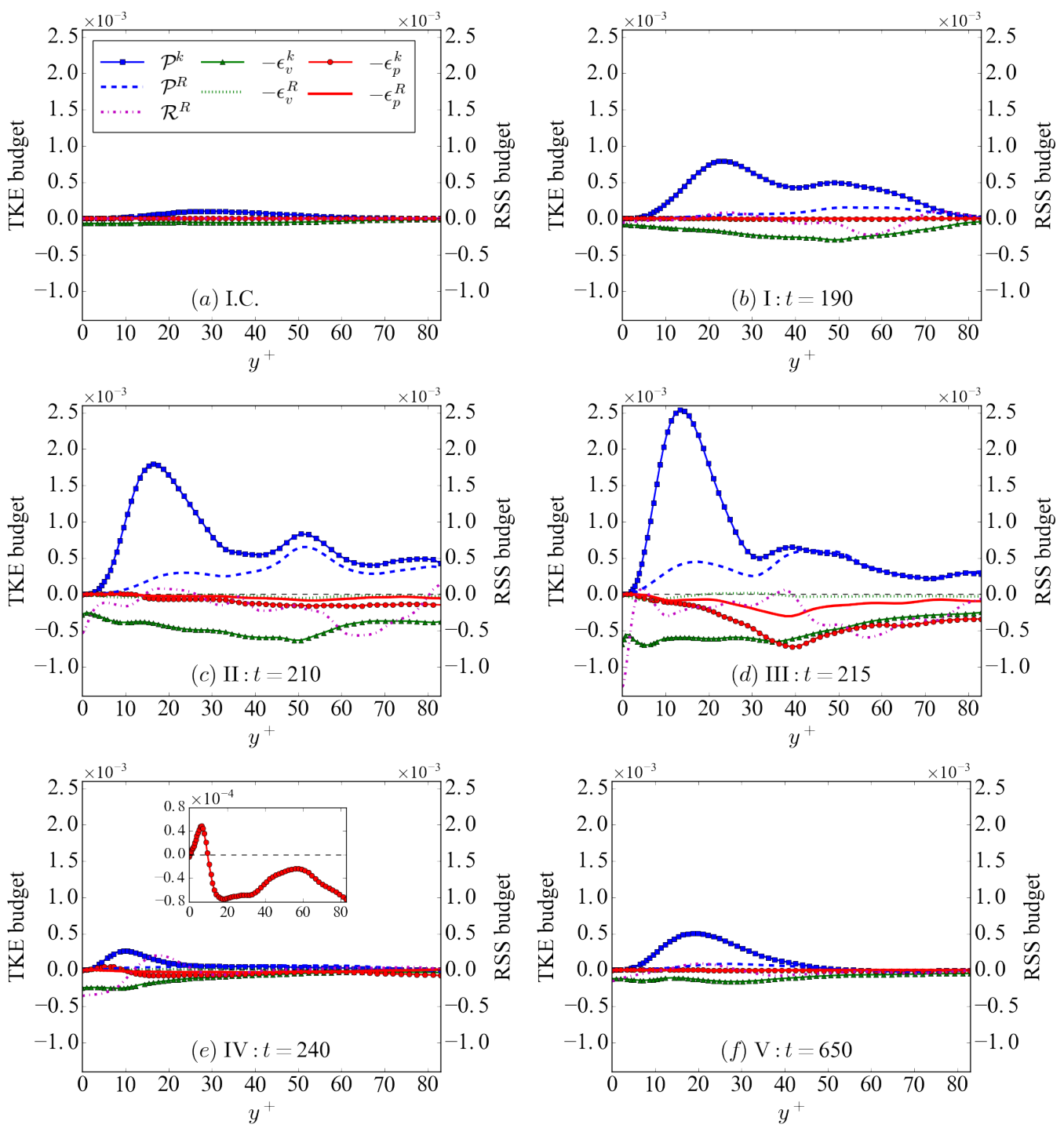

Figure 16: TKE and RSS budgets for the Wi $=28$ case: (a) initial condition; (b)-(f) are are moments $\mathrm{I}-\mathrm{V}$. The inset of panel (e) shows the profile of $-\epsilon_{\mathrm{p}}^{k}$ separately. 
now (moment III) peaks in the buffer layer as well and its profile conforms to that of $\mathcal{P}^{k}$, indicating that the high-intensity small-scale flow structures resulting from the breakdown of primary vortices reinforce the RSS generation (which thus completes a positive feedback loop as the RSS further drives the TKE production). The TKE viscous dissipation $\epsilon_{\mathrm{v}}^{k}$ also suddenly jumps to a comparable magnitude as that of $\mathcal{P}^{k}$, which eventually tames the fluctuations and regulates the eddies into the typical coherent structures of the turbulent SCS. For the RSS budget, $\epsilon_{\mathrm{v}}^{\mathrm{R}}$ is small and $\mathcal{P}^{\mathrm{R}}$ is counteracted instead by $\mathcal{R}^{\mathrm{R}}$.

In the viscoelastic case (fig. $16(\mathrm{~d})$ ), the $\mathcal{P}^{k}$ profile at moment III is still similar in shape as the Newtonian case but its magnitude is much lower and the peak location is farther away from the wall. By contrast, $\mathcal{P}^{\mathrm{R}}$ maintains its flat profile through moment III (in comparison, in the Newtonian case, the profile changes shape and peaks in the buffer layer - see fig. 15(c)). Excessive dissipation $\epsilon_{\mathrm{v}}^{k}$ near the wall, observed in the Newtonian case at moment III, is also avoided. These observations are consistent with the main conclusion so far that adding polymers allows the transition to bypass the strong structural bursting and mark the major difference between the Newtonian and viscoelastic pathways. The polymer elastic conversion terms $-\epsilon_{\mathrm{p}}^{k}$ and $-\epsilon_{\mathrm{p}}^{\mathrm{R}}$ are trivial until moment III, where their magnitudes suddenly surge. Both terms are negative across the channel: polymer interaction with the flow reduces both TKE and RSS and suppresses turbulent motion. In the TKE budget, $-\epsilon_{\mathrm{p}}^{k}$ becomes comparable to the viscous term $-\epsilon_{\mathrm{v}}^{k}$ and both converts mechanical energy from turbulent motions to the internal energy of the fluids. In the RSS budget, $-\epsilon_{\mathrm{p}}^{\mathrm{R}}$ is the main force for balancing the RSS production $\mathcal{P}^{\mathrm{R}}$ and (different from the Newtonian case) $\mathcal{R}^{\mathrm{R}}$ becomes 
less important.

At moment IV, all terms retreat to magnitudes close to their SCS levels (moment V). Interestingly, although $-\epsilon_{\mathrm{p}}^{k}$ remains negative in most of the channel, in regions very close to the wall $\left(y^{+} \lesssim 10\right)$ it becomes positive - polymer elastic energy is being converted to the TKE. In other words, the flow instability is driven, in part, by polymer elasticity. This change in the $-\epsilon_{\mathrm{p}}^{k}$ profile, although small, is not trivial. It is accompanied by a clear change in the flow structure. Direct flow visualization of the same moment was shown in fig. 9(e). Vortices are more densely populated near the top wall, whereas at the bottom side (where the ES originally resided) the distribution is sparse. The image only shows quasi-stremwise vortices typical of IDT. However, smaller patches of spanwise vortices can also be spotted near the bottom wall (co-existing with streamwise vortices) if a lower $Q$ level is used for the isosurface. (They are currently not visible in fig. 9(e) because their vortical strength is much weaker than that of the dominant IDT vortices.) These structures strongly resemble the characteristic structure of EIT [31]. Their occurrence is transient and does not last: they have completely vanished in moment $\mathrm{V}$. The same observations, i.e., positive $-\epsilon_{\mathrm{p}}^{k}$ near the wall and the sporadic and intermittent appearance of EIT-like spanwise vortices alongside the dominant IDT vortices, are found in all three Wi studied. They consistently show up on the channel side of the original ES and in the time period immediately after the bursting - probably as a result of the strong polymer stretching by the intense turbulent structures at moment III. It was discussed earlier that at higher Wi, IDT lasts only for a finite time period and the trajectory may eventually converge to EIT (where spanwise vortices 
fill the domain and quasi-streamwise vortices vanish). The observation here further adds that even in regimes dominated by IDT, EIT-like structures can still co-exist with the dominant vortices. The co-existence of EIT and IDT solutions in the state spaces, as well as the co-existence of two types of vortex structures in the latter, will be a subject of our continued research. Finally, it is noted that the initial turbulence development and its overshoot (moments I, II, \& III) remain inertia-driven and are unaffected by EIT-like structures - the latter appear to be a result of the bursting dynamics rather than its cause.

\section{Conclusions}

The transient problem of turbulence growth from the edge state, its subsequent bursting event, and the polymer effects thereon are studied through dynamical shooting trajectories. In Newtonian flow, the transition starts with a strong overshoot of the Reynolds shear stress which results from the intensification of the streamwise velocity streams as well as the three-dimensional distortion of the primary vortices. As the RSS reaches the maximum, streak instability quickly leads to a breakdown of the primary vortices into a dense cloud of small but high-intensity eddies, at which the turbulent kinetic energy rises to its peak. These small-scale fluctuations also result in a sudden

jump in the viscous dissipation rate, which quenches the fluctuations and regulates the flow into the SCS of turbulence. Introducing drag-reducing polymers does not affect the initial growth of instability. However, it stabilizes the primary streak-vortex structures and prevents the breakdown into small-scale eddies. Significant polymer stress and conversion to elastic en- 
ergy are only observed after the peak of TKE, where breakdown would have happened in Newtonian flow. By design, the shooting trajectories studied here follow the unstable manifold of the ES and track the development of inertia-driven instability. Polymers do not alter the nature of this pathway and only suppress turbulence growth. The lack of polymer effects during the initial growth of turbulence indicates that near the ES there is a state-space region where polymers do not suppress turbulence, which is the qualitative feature of MDR.

A simple dynamical model based on the current knowledge of the statespace solutions describes the statistically-converged turbulent dynamics as a three-stage cycle: (1) the flow samples the center of the turbulent basin, which takes up the majority of turbulence life time in the Newtonian limit and becomes shortened as polymer influence increases [55, 23]; (2) intermittent escape from the this active turbulence brings the system near the ES the so-called hibernating turbulence [55, 23, 54]; (3) as the flow is bounced back by the ES, it follows its unstable manifold and goes through a similar overshoot stage - which appears as bursting events - before returning to active turbulence. Studying the polymer effects on the transient growth of turbulence not only contributes to our knowledge of the bypass transition in viscoelastic fluid flows, it also helps us understand the bursting dynamics in viscoelastic turbulence. In particular, the regulation of bursting by polymers revealed by this study offers a direct explanation for the significant reduction in disturbance redistribution, which according to Zhu et al. [25] is a key mechanism for the LDR-HDR transition. The large magnitudes of polymer shear stress during this process would also explain the conflicting 
observations in previous experimental and numerical studies. Finally, our results also reveal the co-existence of turbulent states driven by fluid elasticity together with that driven by inertia. The current study focuses on the transition dynamics into the inertia-driven turbulence. Future research is required to paint a complete picture of these different types of solution objects in the state space.

\section{Acknowledgments}

The authors gratefully acknowledge the financial support from the Natural Sciences and Engineering Research Council of Canada (NSERC) through its Discovery Grants Program (No. RGPIN-2014-04903) as well as the computing resource allocated by Compute/Calcul Canada. This work is made possible by the facilities of the Shared Hierarchical Academic Research Com-

puting Network (SHARCNET: www.sharcnet.ca). LX also acknowledges the National Science Foundation Grant No. NSF PHY11-25915, which partially supported his stay at the Kavli Institute for Theoretical Physics (KITP) at UC Santa Barbara.

\section{References}

[1] P. S. Virk, Drag reduction fundamentals, AIChE J. 21 (1975) 625-656.

[2] M. D. Graham, Drag reduction in turbulent flow of polymer solutions, in: D. M. Binding, K. Walters (Eds.), Rheology Reviews 2004, British Society of Rheology, 2004, pp. 143-170. 
[3] C. M. White, M. G. Mungal, Mechanics and prediction of turbulent drag reduction with polymer additives, Annu. Rev. Fluid Mech. 40 (2008) $235-256$.

[4] M. D. Graham, Drag reduction and the dynamics of turbulence in simple and complex fluids, Phys. Fluids 26 (2014) 101301. doi:10.1063/1.4895780.

[5] B. A. Toms, Some observations on the flow of linear polymer solutions through straight tubes at large Reynolds numbers, in: Proc. 1st Int'l. Congress on Rheology, volume 2, Amsterdam, 1948, pp. 135-141.

[6] M. D. Warholic, D. K. Heist, M. Katcher, T. J. Hanratty, A study with particles image velocimetry of the influence of drag-reducing polymers on the structure of turbulence, Exp. Fluids 31 (2001) 474-483.

[7] C. M. White, V. S. R. Somandepalli, M. G. Mungal, The turbulence structure of drag-reduced boundary layer flow, Exp. Fluids 36 (2004) 62-69.

[8] R. Sureshkumar, A. N. Beris, R. A. Handler, Direct numerical simulation of the turbulent channel flow of a polymer solution, Phys. Fluids 9 (1997) 743-755.

[9] T. Min, J. Y. Yoo, H. Choi, D. D. Joseph, Drag reduction by polymer additives in a turbulent channel flow, J. Fluid Mech. 486 (2003) 213-238.

[10] E. De Angelis, C. M. Casciola, R. Piva, DNS of wall turbulence: dilute polymers and self-sustaining mechanisms, Comput. Fluids 31 (2002) 495-507. 
[11] Y. Dubief, V. E. Terrapon, C. M. White, E. S. G. Shaqfeh, P. Moin, S. K. Lele, New answers on the interaction between polymers and vortices in turbulent flows, Flow Turbul. Combust. 74 (2005) 311-329.

[12] W. Li, M. D. Graham, Polymer induced drag reduction in exact coherent structures of plane Poiseuille flow, Phys. Fluids 19 (2007) 083101.

[13] K. Kim, C. F. Li, R. Sureshkumar, S. Balachandar, R. J. Adrian, Effects of polymer stresses on eddy structures in dragreduced turbulent channel flow, J. Fluid Mech. 584 (2007) 281-299. doi:10.1017/S0022112007006611.

[14] L. Xi, M. D. Graham, Turbulent drag reduction and multistage transitions in viscoelastic minimal flow units, J. Fluid Mech. 647 (2010) 421-452. doi:10.1017/S0022112010000066.

[15] S. B. Pope, Turbulent flows, Cambridge University Press, Cambridge, United Kingdom, 2000.

[16] C. M. White, Y. Dubief, J. Klewicki, Re-examining the logarithmic dependence of the mean velocity distribution in polymer drag reduced wallbounded flow, Phys. Fluids 24 (2012) 021701. doi:10.1063/1.3681862.

[17] B. R. Elbing, M. Perlin, D. R. Dowling, S. L. Ceccio, Modification of the mean near-wall velocity profile of a high-Reynolds number turbulent boundary layer with the injection of drag-reducing polymer solutions, Phys. Fluids 25 (2013) 085103. doi:10.1063/1.4817073.

[18] K. D. Housiadas, A. N. Beris, R. A. Handler, Viscoelastic effects on 
higher order statistics and on coherent structures in turbulent channel flow, Phys. Fluids 17 (2005) 035106.

[19] C. F. Li, R. Sureshkumar, B. Khomami, Influence of rheological parameters on polymer induced turbulent drag reduction, J. Non-Newton. Fluid Mech. 140 (2006) 23-40.

[20] L. Xi, Nonlinear dynamics and instabilities of viscoelastic fluid flows, Ph.D. thesis, University of Wisconsin-Madison, 2009. URL: http://grahamgroup. che.wisc.edu/pub/LiXi_dissertation.pdf.

[21] K. R. Sreenivasan, C. M. White, The onset of drag reduction by dilute polymer additives, and the maximum drag reduction asymptote, J. Fluid Mech. 409 (2000) 149-164.

[22] I. Procaccia, V. S. L'vov, R. Benzi, Colloquium: theory of drag reduction by polymers in wall-bounded turbulence, Rev. Mod. Phys. 80 (2008) 225-247.

[23] L. Xi, M. D. Graham, Intermittent dynamics of turbulence hibernation in Newtonian and viscoelastic minimal channel flows, J. Fluid Mech. 693 (2012) 433-472. doi:10.1017/jfm.2011.541.

[24] M. D. Warholic, G. M. Schmidt, T. J. Hanratty, The influence of a drag-reducing surfactant on a turbulent velocity field, J. Fluid Mech. 388 (1999) 1-20.

[25] L. Zhu, H. Schrobsdorff, T. M. Schneider, L. Xi, Distinct transition in flow statistics and vortex dynamics between low- and high-extent 
turbulent drag reduction in polymer fluids, J. Non-Newton. Fluid Mech. 262 (2018) 115-130. doi:10.1016/j.jnnfm.2018.03.017.

[26] W. B. Giles, W. T. Pettit, Stability of dilute viscoelastic flows, Nature 216 (1967) 470-472. doi:10.1038/216470a0.

[27] A. A. Draad, G. D. C. Kuiken, F. T. M. Nieuwstadt, Laminar-turbulent transition in pipe flow for Newtonian and non-Newtonian fluids, J. Fluid Mech. 377 (1998) 267-312. doi:10.1017/S0022112098003139.

[28] M. P. Escudier, F. Presti, S. Smith, Drag reduction in the turbulent pipe flow of polymers, J. Non-Newton. Fluid Mech. 81 (1999) 197-213. doi:10.1016/S0377-0257(98)00098-6.

[29] A. Ram, A. Tamir, Structural turbulence in polymer solutions, J. Appl. Polym. Sci. 8 (1964) 2751-2762. doi:10.1002/app.1964.070080621.

[30] R. J. Hansen, R. C. Little, Early turbulence and drag reduction phenomena in larger pipes, Nature 252 (1974) 690. doi:10.1038/252690a0.

[31] D. Samanta, Y. Dubief, M. Holzner, C. Schäfer, A. N. Morozov, C. Wagner, B. Hof, Elasto-inertial turbulence, Proc. Natl. Acad. Sci. U. S. A. 110 (2013) 10557-100562. doi:10.1073/pnas.1219666110.

[32] G. H. Choueiri, J. M. Lopez, B. Hof, Exceeding the asymptotic limit of polymer drag reduction, Phys. Rev. Lett. 120 (2018) 124501. doi:10.1103/PhysRevLett.120.124501.

[33] S. Sid, V. E. Terrapon, Y. Dubief, Two-dimensional dynamics of elasto- 
inertial turbulence and its role in polymer drag reduction, Phys. Rev. Fluids 3 (2018) 011301. doi:10.1103/PhysRevFluids.3.011301.

[34] J. Jimenez, Bifurcations and bursting in two-dimensional Poiseuille flow, Phys. Fluids 30 (1987) 3644-3646. doi:10.1063/1.866450.

[35] M. Nishioka, M. Asai, Some observations of the subcritical transition in plane poiseuille flow, J. Fluid Mech. 150 (1985) 441-450.

[36] R. Jacobs, P. Durbin, Simulations of bypass transition, J. Fluid Mech. 428 (2001) 185-212. doi:10.1017/S0022112000002469.

[37] P. Durbin, X. Wu, Transition beneath vortical disturbances, Annu. Rev. Fluid Mech. 39 (2007) 107-128. doi:10.1146/annurev.fluid.39.050905.110135.

[38] D. Henningson, A. Lundbladh, A. Johansson, A mechanism for bypass transition from localized disturbances in wall-bounded shear flows, J. Fluid Mech. 250 (1993) 169-207. doi:10.1017/S0022112093001429.

[39] T. Zaki, P. Durbin, Mode interaction and the bypass route to transition, J. Fluid Mech. 531 (2005) 85-111. doi:10.1017/S0022112005003800.

[40] P. Schlatter, L. Brandt, H. C. de lange, D. S. Henningson, On streak breakdown in bypass transition, Phys. Fluids 20 (2008) 101505. doi:10.1063/1.3005836.

[41] X. Wu, P. Moin, J.-P. Hickey, Boundary layer bypass transition, Phys. Fluids 26 (2014) 091104. doi:10.1063/1.4893454. 
[42] X. Wu, P. Moin, R. J. Adrian, J. R. Baltzer, Osborne Reynolds pipe flow: Direct simulation from laminar through gradual transition to fully developed turbulence, Proc. Natl. Acad. Sci. U. S. A. 112 (2015) 79207924. doi:10.1073/pnas.1509451112.

[43] N. Hoda, M. R. Jovanovic, S. Kumar, Energy amplification in channel flows of viscoelastic fluids, J. Fluid Mech. 601 (2008) 407-424. doi:10.1017/S0022112008000633.

[44] N. Hoda, M. R. Jovanovic, S. Kumar, Frequency responses of streamwise-constant perturbations in channel flows of Oldroyd-B fluids, J. Fluid Mech. 625 (2009) 411-434. doi:10.1017/S0022112009006223.

[45] M. Zhang, I. Lashgari, T. A. Zaki, L. Brandt, Linear stability analysis of channel flow of viscoelastic Oldroyd-B and FENE-P fluids, J. Fluid Mech. 737 (2013) 249-279. doi:10.1017/jfm.2013.572.

[46] J. Page, T. A. Zaki, Streak evolution in viscoelastic Couette flow, J. Fluid Mech. 742 (2014) 520-551. doi:10.1017/jfm.2013.686.

[47] A. Agarwal, L. Brandt, T. A. Zaki, Linear and nonlinear evolution of a localized disturbance in polymeric channel flow, J. Fluid Mech. 760 (2014) 278-303. doi:10.1017/jfm.2014.586.

[48] J. F. Gibson, J. Halcrow, P. Cvitanotić, Visualizing the geometry of state-space in plane Couette flow, J. Fluid Mech. 611 (2008) 107-130.

[49] P. Cvitanotić, Recurrent flows: the clockwork behind turbulence, J. Fluid Mech. 726 (2013) 1-4. doi:10.1017/jfm.2013.198. 
[50] J. D. Skufca, J. A. Yorke, B. Eckhardt, Edge of chaos in a parallel shear flow, Phys. Rev. Lett. 96 (2006) 174101.

[51] T. M. Schneider, B. Eckhardt, J. A. Yorke, Turbulence transition and the edge of chaos in pipe flow, Phys. Rev. Lett. 99 (2007) 034502.

[52] T. Khapko, T. Kreilos, P. Schlatter, Y. Duguet, B. Eckhardt, D. S. Henningson, Edge states as mediators of bypass transition in boundarylayer flows, J. Fluid Mech. 801 (2016) R2. doi:10.1017/jfm.2016.434.

[53] L. Xi, M. D. Graham, Dynamics on the laminar-turbulent boundary and the origin of the maximum drag reduction asymptote, Phys. Rev. Lett. 108 (2012) 028301. doi:10.1103/PhysRevLett.108.028301.

[54] L. Xi, X. Bai, Marginal turbulent state of viscoelastic fluids: A polymer drag reduction perspective, Phys. Rev. E 93 (2016). doi:10.1103/PhysRevE.93.043118.

[55] L. Xi, M. D. Graham, Active and hibernating turbulence in minimal channel flow of Newtonian and polymeric fluids, Phys. Rev. Lett. 104 (2010) 218301. doi:10.1103/PhysRevLett.104.218301.

[56] S. N. Wang, M. D. Graham, F. J. Hahn, L. Xi, Time-series and extended Karhunen-Loève analysis of turbulent drag reduction in polymer solutions, AIChE J. 60 (2014) 1460-1475. doi:10.1002/aic.14328.

[57] J. S. Park, M. D. Graham, Exact coherent states and connections to turbulent dynamics in minimal channel flow, J. Fluid Mech. 782 (2015) 430-454. doi:10.1017/jfm.2015.554. 
[58] J. Wang, J. F. Gibson, F. Waleffe, Lower branch coherent states in shear flows: transition and control, Phys. Rev. Lett. 98 (2007) 204501.

[59] R. D. Whalley, J. S. Park, A. Kushwaha, D. J. C. Dennis, M. D. Graham, R. J. Poole, Low-drag events in transitional wall-bounded turbulence, Phys. Rev. Fluids 2 (2017) 034602. doi:10.1103/PhysRevFluids.2.034602.

[60] A. Kushwaha, J. S. Park, M. D. Graham, Temporal and spatial intermittencies within channel flow turbulence near transition, Phys. Rev. Fluids 2 (2017) 024603. doi:10.1103/PhysRevFluids.2.024603.

[61] S. K. Robinson, Coherent motions in the turbulent boundary layer, Annu. Rev. Fluid Mech. 23 (1991) 601-639.

[62] J. Jiménez, G. Kawahara, M. P. Simens, M. Nagata, M. Shiba, Characterization of near-wall turbulence in terms of equilibrium and "bursting" solutions, Phys. Fluids 17 (2005) 015105.

[63] W. D. McComb, L. H. Rabie, Drag-reducing polymers and turbulent bursts, Nature 273 (1978) 653-654. doi:10.1038/273653a0.

[64] L. Xi, M. Shah, B. L. Trout, Hopping of water in a glassy polymer studied via transition path sampling and likelihood maximization, J. Phys. Chem. B 117 (2013) 3634-3647. doi:10.1021/jp3099973.

[65] R. B. Bird, C. F. Curtis, R. C. Armstrong, O. Hassager, Dynamics of polymeric liquids, volume 2, 2nd ed., John Wiley \& Sons, New York, 1987. 
[66] J. Jiménez, P. Moin, The minimal flow unit in near-wall turbulence, J. Fluid Mech. 225 (1991) 213-240.

[67] Y. Duguet, P. Schlatter, D. S. Henningson, Localized edge states in plane couette flow, Phys. Fluids 21 (2009) 111701.

[68] T. M. Schneider, D. Marinc, B. Eckhardt, Localized edge states nucleate turbulence in extended plane Couette cells, J. Fluid Mech. 646 (2010) $441-451$.

[69] R. Peyret, Spectral methods for incompressible viscous flow, Springer, New York, 2002.

[70] D. Zhang, C. Jiang, D. Liang, L. Cheng, A review on tvd schemes and a refined flux-limiter for steady-state calculations, J. Comput. Phys. 302 (2015) 114-154.

[71] B. Yu, Y. Kawaguchi, Direct numerical simulation of viscoelastic dragreducing flow: a faithful finite difference method, J. Non-Newton. Fluid Mech. 116 (2004) 431-466. doi:10.1016/j.jnnfm.2003.11.006.

[72] R. Sureshkumar, A. N. Beris, Effect of artificial stress diffusivity on the stability of numerical calculations and the flow dynamics of timedependent viscoelastic flows, J. Non-Newton. Fluid Mech. 60 (1995) 53-80. doi:10.1016/0377-0257(95)01377-8.

[73] P. K. Ptasinski, B. J. Boersma, F. T. M. Nieuwstadt, M. A. Hulsen, B. H. A. A. van den Brule, J. C. R. Hunt, Turbulent channel flow near maximum drag reduction: simulations, experiments and mechanisms, J. Fluid Mech. 490 (2003) 251-291. 
[74] K. D. Housiadas, A. N. Beris, Polymer-induced drag reduction: effects of variations in elasticity and inertia in turbulent viscoelastic channel flow, Phys. Fluids 15 (2003) 2369-2384.

[75] C. F. Li, R. Sureshkumar, B. Khomami, Simple framework for understanding the universality of the maximum drag reduction asymptote in turbulent flow of polymer solutions, Phys. Rev. E 92 (2015) 043014. doi:10.1103/PhysRevE.92.043014.

[76] L. Zhu, L. Xi, Coherent structure dynamics and identification during the multistage transitions of polymeric turbulent channel flow, J. Phys.: Conf. Ser. 1001 (2018) 012005. doi:10.1088/1742-6596/1001/1/012005.

[77] A. W. El-Kareh, L. G. Leal, Existence of solutions for all deborah numbers for a non-newtonian model modified to include diffusion, J. Non-Newton. Fluid Mech. 33 (1989) 257-287. doi:10.1016/03770257(89)80002-3.

[78] T. Vaithianathan, A. Robert, J. G. Brasseur, L. R. Collins, An improved algorithm for simulating three-dimensional, viscoelastic turbulence, Journal of non-newtonian fluid mechanics 140 (2006) 3-22. doi:10.1016/j.jnnfm.2006.03.018.

[79] T. Min, J. Y. Yoo, H. Choi, Effect of spatial discretization schemes on numerical solutions of viscoelastic fluid flows, J. Non-Newton. Fluid Mech. 100 (2001) 27-47. doi:10.1016/S0377-0257(01)00128-8.

[80] S. He, M. Seddighi, Transition of transient channel flow after a 
change in Reynolds number, J. Fluid Mech. 764 (015) 395-427. doi:10.1017/jfm.2014.698.

[81] S. Toh, T. Itano, A periodic-like solution in channel flow, J. Fluid Mech. 481 (2003) 67-76.

[82] T. M. Schneider, J. F. Gibson, M. Lagha, F. De Lillo, B. Eckhardt, Laminar-turbulent boundary in plane Couette flow, Phys. Rev. E 78 (2008) 037301. doi:10.1103/PhysRevE.78.037301.

[83] Y. Duguet, A. P. Willis, R. R. Kerswell, Transition in pipe flow: the saddle structure on the boundary of turbulence, J. Fluid Mech. 613 (2008) 255-274.

[84] G. A. Webber, R. A. Handler, L. Sirovich, The Karhunen-Loéve decomposition of minimal channel flow, Phys. Fluids 9 (1997) 1054-1066.

[85] C. R. Smith, S. P. Metzler, The characteristics of low-speed streaks in the near-wall region of a turbulent boundary-layer, J. Fluid Mech. 129 (1983) 27-54.

[86] J. Jeong, F. Hussain, On the identification of a vortex, J. Fluid Mech. 285 (1995) 69-94.

[87] N. R. Lebovitz, Boundary collapse in models of shear-flow transition, Commun. Nonlinear Sci. Numer. Simul. 17 (2012) 2095-2100. doi:10.1016/j.cnsns.2011.07.023.

[88] J. Kim, P. Moin, R. Moser, Turbulence statistics in fully-developed 
channel flow at low Reynolds-number, J. Fluid Mech. 177 (1987) 133166.

[89] M. D. Warholic, H. Massah, T. J. Hanratty, Influence of drag-reducing polymers on turbulence: effects of Reynolds number, concentration and mixing, Exp. Fluids 27 (1999) 461-472.

[90] T. Min, H. Choi, J. Y. Yoo, Maximum drag reduction in a turbulent channel flow by polymer additives, J. Fluid Mech. 492 (2003) 91-100. 